\title{
Forward Scattering Approximation and Bosonization in Integer Quantum Hall Systems
}

\author{
M. Rosenau da Costa, ${ }^{1,2}$ H. Westfahl Jr., ${ }^{3}$ and A.O. Caldeira ${ }^{2}$ \\ ${ }^{1}$ International Center of Condensed Matter Physics, Universidade de Brasília, Caixa Postal 04667, 70910-900, Brasília-DF, Brazil \\ ${ }^{2}$ Instituto de Física "Gleb Wataghin”, Universidade Estadual de Campinas, Caixa Postal 6165, 13083-970, Campinas-SP, Brazil \\ ${ }^{3}$ Laboratório Nacional de Luz Síncrotron - ABTLuS, Caixa Postal 6192, 13043-090, Campinas-SP, Brazil
}

(Dated: September 25, 2018)

\begin{abstract}
In this work we present a model and a method to study integer quantum Hall (IQH) systems. Making use of the Landau levels structure we divide these two dimensional systems into a set of interacting one dimensional gases, one for each guiding center. We show that the so-called strong field approximation, used by Kallin and Halperin and by MacDonald, is equivalent, in first order, to a forward scattering approximation and analyze the IQH systems within this approximation. Using an appropriate variation of the Landau level bosonization method we obtain the dispersion relations for the collective excitations and the single particle spectral functions. For the bulk states, these results evidence a behavior typical of non-normal strongly correlated systems, including the spin-charge splitting of the single particle spectral function. We discuss the origin of this behavior in the light of the Tomonaga-Luttinger model and the bosonization of two dimensional electron gases.
\end{abstract}

PACS numbers: 71.10.Pm, 71.70.Di, 73.43.-f, 73.43.Cd, 73.43.Lp

\section{INTRODUCTION}

The integer quantum Hall effect (IQHE) $\frac{1}{\underline{1}}$ appears in two dimensional electron gases (2DEG) under a strong magnetic field, $B$, with the electronic Landau level filling factor, $v$, equals to an integer number. In this case the non-interacting ground state presents $v$ completely filled Landau levels and the excitons generated by the electron-electron interaction involve particles in different levels. At stronger magnetic fields the degeneracy, $N_{\phi}=S / 2 \pi l^{2}$ (where $S$ is the area of the 2DEG and $l=\sqrt{c \hbar /(e B)}$ is the magnetic length), of a single Landau level exceeds the number of electrons. In this case many non-interacting ground states can be constructed with all the electrons in the macroscopically degenerate lowest Landau level. In this case the usual perturbative theories, which lead to the Fermi Liquid behavior, are invalid and the effect of the electron-electron interaction turns out to be much more dramatic, giving rise to a new state of the matter and to the fractional quantum Hall effect (FQHE) $)^{2.3}$.

The study of collective excitations plays an important role in understanding both systems. The existence of magnetorotons ${ }^{4.5 .6}$ was verified through inelastic light scattering ${ }^{7}$ and phonon spectroscopy ${ }^{8,9}$. However, in the integer quantum Hall systems, at very low electronic densities (less than $10^{-10} \mathrm{~cm}^{-2}$ ) multiple magneto-rotons which are not accessible to the usual perturbative theories (possibly because they neglect the mixing of the Landau levels) were observed 10 , demonstrating an incomplete understanding of these excitations. Additionally, in the FQHE the coupling between the particle excitations with the magneto-plasmon mode close to the cyclotron frequency, $\omega_{c}$, seems to be the key for a Hamiltonian theory of these systems 11 , and to the origin of most part of the notable properties of their quasi-particles, the composite fermions 12,13 . Here we will show that the collective excitations also seem to have a strong influence in the bulk quasi-particles properties even in the IQHE.

In this work we will explicitly treat the integer quantum Hall systems, although the method we present, together with the Chern-Simons theory ${ }^{14}$, also has applications in the frac- tional case ${ }^{15}$. Assuming a system where the Coulomb energy, $e^{2} / \varepsilon l$, is much smaller than the cyclotron energy, $\hbar \omega_{c}=$ $\hbar e B /(m c)$, the so-called strong field approximation ${ }^{4.5}$ (where the mixing between the Landau levels is neglected) can be used. Under this approximation, the collective magnetoplasma modes were determined by the Green's function method $^{4}$ and time-dependent Hartree-Fock theory ${ }^{5}$. In this work, we will see that these modes can also be obtained by a magneto-exciton bosonization method. First, making use of the natural structure of the Landau levels, we divide the 2DEG into a set of $N_{\phi}$ one dimensional interacting channels, one for each guiding center occupied by $v$ particles. We show that, to first order in $\left(e^{2} / \varepsilon l\right) / \hbar \omega_{c}$, the strong field approximation is equivalent to a forward scattering approximation in this model of one dimensional interacting channels. Thus, we will allow for the transfer of energy and momentum between the different channels mediated by the direct and exchange Coulomb interactions, conserving the net number of particles in each channel. We then use a variation of the Landau level bosonization ${ }^{16}$ which is ideal for a treatment of the interaction effects in this model.

One of the major advantages of this bosonization method is that, through the Mattis-Mandelstam bosonic representation of the fermionic operator ${ }^{17,18}$, one is be able to determine the single particle properties directly from the collective modes, differently from the usual perturbative theory. Another advantage is that this Mattis-Mandelstam representation is an identity 18 which, technicaly, can be used later to extend the model, including the net transfer of particles between neighbor channels according to their relative angular momentum. We believe that bosonization procedures similar to this one can be important tools to clarify the relation between the quasi-particle properties and the collective modes in the FQHE. Indeed, the Hamiltonian Theory of the FQHE 11 is very similar to a bosonization of the degrees of freedom related to the magneto-plasmon excitation.

The organization of the paper is as follows. In section II we review the free and the interacting Hamiltonians of a two dimensional electron gas in a perpendicular magnetic field writ- 
ten in the Landau level basis and in the symmetric gauge. In section III we show the equivalence between the strong field approximation and the forward scattering approximation and then describe how to treat the 2DEG, including the direct and exchange interactions between the electrons in a bosonization approach. In section IV we present a comparative analysis of our bosonized model with the Tomonaga-Luttinger model and the 2D bosonization. Next we describe the strategy to deal with spin-full fermions in section V. The spectrum of neutral excitations obtained is presented in section VI. The construction of the fermionic operator and the determination of the single-particle spectral functions is accomplished in section VII and our conclusions are presented in section VIII.

\section{FERMIONIC REPRESENTATION}

We consider a 2 DEG in a strong perpendicular magnetic field at zero temperature with an integer number, $v$, of fully occupied Landau levels. We start from a simpler description of spinless electrons and generalize the model to the case of electrons with spins in section VII.

The non-interacting part of the Hamiltonian of spinless fermions of mass $m$ in a plane perpendicular to an uniform magnetic field is given by

$$
H_{0}=\frac{1}{2 m} \int d^{2} \mathbf{r} \Psi^{\dagger}(\mathbf{r})\left[\mathbf{p}-\frac{e}{c} \mathbf{A}(\mathbf{r})\right]^{2} \Psi(\mathbf{r}),
$$

where $\Psi(\mathbf{r})$ is the fermion field operator, which can be expanded in the Landau level basis and in the symmetric gauge, $\mathbf{A}(\mathbf{r})=\frac{B}{2} \hat{z} \times \mathbf{r}$, as

$$
\Psi(\mathbf{r})=\sum_{n, m}\langle\mathbf{r} \mid n, m\rangle c_{n, m}
$$

Here $|n, m\rangle$ are the eigenstates of the one particle Hamiltonian, where $n$ refers to the Landau level and $m$ to the guiding center, with 21

$$
\langle\mathbf{r} \mid n, m\rangle=\frac{e^{-z^{2} / 4 l^{2}}}{\sqrt{2 \pi l^{2}}} G_{m, n}\left(i \frac{\mathbf{z}^{*}}{l}\right),
$$

where $\mathbf{z}=x+i y$. For $m>n$,

$$
G_{m, n}(\mathbf{z})=\sqrt{\frac{n !}{m !}}\left(\frac{-i \mathbf{z}}{\sqrt{2}}\right)^{m-n} L_{n}^{m-n}\left(\frac{z^{2}}{2}\right),
$$

with $L_{n}^{m-n}$ being the generalized Laguerre polynomial. The Hamiltonian $H_{0}$ is diagonal in this Landau levels basis

$$
H_{0}=\hbar \omega_{c} \sum_{m=0}^{N_{\Phi}-1} \sum_{n=0}^{\infty}(n+1 / 2) c_{n, m}^{\dagger} c_{n, m} .
$$

Given the guiding center degeneracy, $N_{\phi}$, the non-interacting ground state is defined by uniformly filling $v$ Landau levels of each guiding center $m$ :

$$
\left|G_{\mathbf{0}}\right\rangle=\prod_{m=0}^{N_{\phi}-1} \prod_{n=0}^{v-1} c_{n, m}^{\dagger}|0\rangle
$$

where $N_{\phi}=S / 2 \pi l^{2}$ is the number of flux quanta crossing the area of the system, $S$. The neutral excitations in the noninteracting system are electron-hole pairs with a hole in a Landau level $p \leq v-1$ at the guiding center $m$ and an electron in a level $p+n>v-1$ at the guiding center $m^{\prime}$.

In the Landau levels basis the Coulomb interaction, $V(r)=$ $e^{2} / \varepsilon r$, is formally given by

$$
\begin{aligned}
H_{I}= & \frac{1}{2} \sum_{\{n, m\}}\left\langle n_{1}, m_{1} ; n_{2}, m_{2}|V| n_{1}^{\prime}, m_{1}^{\prime} ; n_{2}^{\prime}, m_{2}^{\prime}\right\rangle \\
& \times c_{n_{1}, m_{1}}^{\dagger} c_{n_{2}, m_{2}}^{\dagger} c_{n_{2}^{\prime}, m_{2}^{\prime}} c_{n_{1}^{\prime}, m_{1}^{\prime}},
\end{aligned}
$$

where the interaction matrix element can be written in the momentum representation as

$$
\begin{aligned}
\left\langle n_{1}, m_{1} ; n_{2}, m_{2}|V| n_{1}^{\prime}, m_{1}^{\prime} ; n_{2}^{\prime}, m_{2}^{\prime}\right\rangle= & \frac{1}{(2 \pi)^{2}} \int d^{2} q \tilde{V}(q) e^{-l^{2} q^{2}} \\
& \times G_{n_{1}^{\prime}, n_{1}}^{*}\left(l \mathbf{q}^{*}\right) G_{m_{1}^{\prime}, m_{1}}^{*}(l \mathbf{q}) \\
& \times G_{n_{2}, n_{2}^{\prime}}\left(l \mathbf{q}^{*}\right) G_{m_{2}, m_{2}^{\prime}}(l \mathbf{q}),
\end{aligned}
$$

with $\tilde{V}(q)=2 \pi e^{2} / \varepsilon q$ and $G$ defined in (2.4).

\section{THE FORWARD SCATTERING APPROXIMATION AND THE BOSONIC DESCRIPTION}

\section{A. First Order Equivalence Between the Strong Field Approximation and the Forward Scattering Approximation}

Inspired by the linear energy dispersion of the quantum number $n$ and the degeneracy of the quantum number $m$, one can make an analogy between the quantum Hall system and the problem of many interacting one-dimensional electron gases. In fact, the elementary neutral excitations spectrum in the integer quantum Hall regime, consisting of electronhole pairs excited over a completely filled set of Landau levels, has been calculated using the diagrammatic Green's function approach of Kallin and Halperin ${ }^{4}$ and the time-dependent Hartree-Fock approximation of MacDonald ${ }^{5}$. In both methods the excited states are constructed essentially in a single mode approximation: (i) considering only the presence of a single electron-hole pair at a time, (ii) over a ground state that neglects any Landau level mixing. Under these approximations, named strong field approximation, the magnetoplasmon bands were calculated including different order terms in the interaction strength, $\gamma=\left(e^{2} / \varepsilon l\right) / \hbar \omega_{c} \ll 1$. Kallin and Halperin include only some first order terms, not all of them, once they do not consider the ones associated with the decay of one exciton into two. MacDonald includes some second order terms, although also excluding the first order ones neglected by Kallin and Halperin. The effect of the decay of excitons was partially considered by Cheng ${ }^{19}$. In Fig.(1), which was constructed according to the conditions (i) and (ii) above, we show that, to first order in $\gamma$, the strong field approximation is analogous to the forward scattering approximation in a set of one-dimensional electron gases, where the net number of particles in each channel (here the guiding center $m$ ) 
is conserved. Indeed, due to the conservation of the angular momentum, the forward scattering approximation will be always restricted to first order effects in $\gamma$, as we discuss in Sec. (III.C). But, as we stressed above, this is not a very restrictive condition, because the previous methods of calculation are not even able to include all these first order effects.

We saw above that if we formally divide a two dimensional IQH system into $m$ interacting one dimensional channels, the strong magnetic field turns the neglect of the net transfer of particles between the " $m$ " channels into a reasonable approximation. So, this system looks very convenient for the use of a bosonization technique, once we can skirt the treatment of the transfer of particles between many different one dimensional channels in this first approximation. The latter is known to be the main difficulty with the higher dimensional bosonization methods ${ }^{20,22}$. However, despite the problem with the many channels particle transfer, there are many techniques developed to study the effects of the net transfer of particles between two channels in a bosonization method. This problem is analogous to the inclusion of the backward scattering in the usual bosonization of a one-dimensional interacting electronic system, which makes the transfer of particles between the right- and left-moving channels ${ }^{41}$. So, in a future work, we intend to included the transfer between two neighbors " $m$ " channels. This neighbor transfer seems to us a promising alternative expansion parameter to the interaction strength $\gamma$, as we discuss further in the conclusion. However, in this work, we will restrict ourselves to the first order terms in the strong field approximation or, equivalently, to the forward scattering approximation.

\section{B. The Bosonic Description}

Motivated by the above discussion, we will introduce bosonic operators associated with electron-hole excitations that conserve $m$, as in the bosonization scheme previously developed by two of us ${ }^{16}$. If we extend the Hilbert space of all guiding centers to negative energy values, in the spirit of the Luttinger model ${ }^{18,24}$, we get that the operator

$$
b_{n, m}^{\dagger}=\frac{1}{\sqrt{n}} \sum_{p=-\infty}^{\infty} c_{n+p, m}^{\dagger} c_{p, m},
$$

and its Hermitian conjugate obey exact bosonic relations:

$$
\left[b_{n, m}, b_{n^{\prime}, m^{\prime}}^{\dagger}\right]=\delta_{m, m^{\prime}} \delta_{n, n^{\prime}}, \quad\left[b_{n, m}, b_{n^{\prime}, m^{\prime}}\right]=0 .
$$

This extension is a well controlled approximation as long as the negative energy states are kept inert. So, the system's Fermi energy must be much higher than the characteristic Coulomb interaction, $v \hbar \omega_{c} \gg e^{2} / \varepsilon l$, which is essentially the strong field approximation.

The Hamiltonian (2.5) can be rewritten in terms of the previously defined bosonic operators as $\frac{18}{18}$

$$
H_{0}=\hbar \omega_{c} \sum_{m=0}^{N_{\Phi}-1}\left[\sum_{n>0} n b_{n, m}^{\dagger} b_{n, m}+\frac{1}{2} \hat{N}_{m}\left(\hat{N}_{m}+1\right)\right],
$$

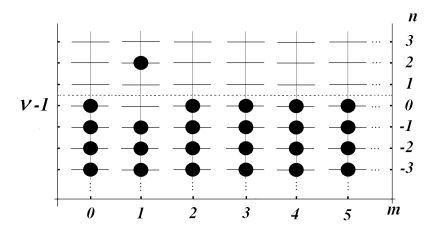

(a)

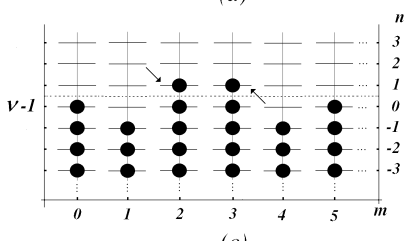

(c)

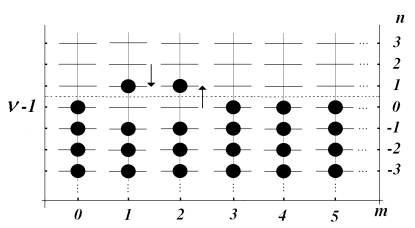

(b)

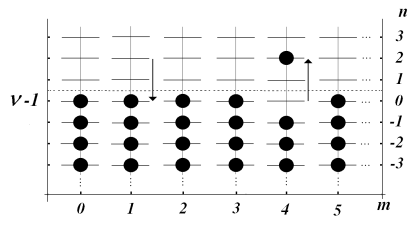

(d)
Figure 1: (a) A single exciton created over a ground state that completely fills the $v-1$ lowest Landau levels, neglecting any Landau level mixing (ii). Consider now only first order processes, where the total energy is conserved, $\sum_{i} \Delta n_{i}=0$. If we neglect multiexciton states (i), processes like (b) or (c), which conserve the total angular momentum in the symmetric gauge, $\Delta L_{z}=\sum_{i}\left(\Delta n_{i}-\Delta m_{i}\right)=0$, will not be allowed. The only dynamical process allowed for this exciton (in first order and constrained to (i) and (ii)) is to decay in its own guiding center and create another exciton in a different one, as in (d). So, the number of particles in each guiding center $m$ will be kept constant. The dispersion curves that we will obtain for the excitons are related to the breakdown of degeneracy between states like (a) and (d). Although the forward scattering approximation allows the decay processes like (b), we will not consider then due to an additional approximation made in the bosonization method.

where $\hat{N}_{m}=\sum_{p=-\infty}^{\infty}\left(c_{p, m}^{\dagger} c_{p, m}-\left\langle G_{\mathbf{0}}\left|c_{p, m}^{\dagger} c_{p, m}\right| G_{\mathbf{0}}\right\rangle\right)$ is the operator which counts the number of particles relative to the reference state $\left|G_{\mathbf{0}}\right\rangle$ in the channel $m$. The last term gives the ground state energy $\left\langle G_{\mathbf{N}}\left|H_{0}\right| G_{\mathbf{N}}\right\rangle$ of a system with a distribution of particles per channel given by the vector $\mathbf{N}=\left(N_{0}, \ldots, N_{\phi-1}\right)$ and the first one describes the excitations above this state. Formally, what allows us to rewrite $(2.5)$ in this alternative bosonic representation, without a further approximation, is the convenient linear dispersion relation in $n$ of the system 18 .

If we now include the Coulomb interaction term (2.6) in the forward scattering approximation and restrict ourselves to the spectrum of low energy excitations (close to the highest filled, $v-1$, Landau level) the total Hamiltonian can be expressed in terms of the bosonic operators $b_{n, m}^{(\dagger)}$, eq. 3.1). Even restricting to processes that conserve the total number of electrons in each guiding center, as in the one dimensional case ${ }^{25}$, there is still more than one way to pair the fermionic operators in 2.6 to yield the bosonic bilinear terms. If we set $m_{1}=m_{1}^{\prime}$ and $m_{2}=m_{2}^{\prime}$ in 2.6 we get the direct scattering processes whereas exchange scattering processes are obtained for $m_{1}=m_{2}^{\prime}$ and $m_{2}=m_{1}^{\prime}$. This leads us to

$H_{I}=\frac{e^{2}}{\varepsilon l} \sum_{n>0, m, m^{\prime}}\left[g_{d i}\left(n ; m, m^{\prime}\right)+g_{e x}\left(n ; m, m^{\prime}\right)\right] b_{n, m}^{\dagger} b_{n, m^{\prime}}+H_{N}$ 
with

$$
\begin{aligned}
g_{d i}\left(n ; m, m^{\prime}\right)= & n \int d \bar{q} \Phi_{m}(\bar{q}) \Phi_{m^{\prime}}(\bar{q}) \tilde{V}_{d i}^{n}(\bar{q}) \\
g_{e x}\left(n ; m, m^{\prime}\right)= & E_{S e l f}^{n} \delta_{m, m^{\prime}} \\
& -n \int d \bar{q} \Phi_{m}(\bar{q}) \Phi_{m^{\prime}}(\bar{q}) \tilde{V}_{e x}^{n}(\bar{q})
\end{aligned}
$$

where $\bar{q}=l q, \Phi_{m}(\bar{q})=\sqrt{\bar{q}} G_{m, m}\left(\frac{\bar{q}^{2}}{2}\right) e^{-\bar{q}^{2} / 4}$,

$$
\begin{aligned}
\tilde{V}_{d i}^{n}(\bar{q})= & \frac{e^{-\bar{q}^{2} / 2}}{\bar{q}}\left|G_{v-1, v-1+n}(\overline{\mathbf{q}})\right|^{2} \\
E_{\text {Self }}^{n}= & \int d \bar{r} e^{-\frac{\bar{r}^{2}}{2}} L_{v-1}^{1}\left(\frac{\bar{r}^{2}}{2}\right)\left[L_{v-1}^{0}\left(\frac{\bar{r}^{2}}{2}\right)\right. \\
& \left.-L_{v-1+n}^{0}\left(\frac{\bar{r}^{2}}{2}\right)\right] \\
\tilde{V}_{e x}^{n}(\bar{q})= & \int d \bar{k} e^{-\frac{\bar{k}^{2}}{2}} L_{v-1+n}\left(\frac{\bar{k}^{2}}{2}\right) L_{v-1}\left(\frac{\bar{k}^{2}}{2}\right) \mathcal{J}_{0}(\bar{k} \bar{q})
\end{aligned}
$$

$\bar{r}=r / l$ and $g_{0}(x)$ is the zero order Bessel function.

In the above expressions, $g_{d i}\left(n ; m, m^{\prime}\right)$ is the contribution (see Appendix B for details) from the direct interaction process 4,6 , that describes the recombination and destruction of the electron-hole pair, and $g_{e x}\left(n ; m, m^{\prime}\right)$ is the contribution from the exchange process (see Appendix B for details). Moreover, $g_{e x}\left(n ; m, m^{\prime}\right)$ describes the bound state of a hole in the level $v-1$ and an electron in the level $v-1+n$ moving along different guiding centers ${ }^{4}$. This is the quantum analogue of the classical interaction of two particles of opposite charge under a magnetic field, which move parallel to one another with a constant velocity perpendicular to their separation. This term has a constant diagonal contribution $E_{S e l f}^{n}$ that is the difference between the exchange self-energy of an electron in the in the excited level $v-1+n$ and the level $v-1$ from which it was removed. Besides, we see that the forward scattering approximation does not generate a diagonal interaction in the guiding centers $m$.

The term $H_{N}$ in (3.4) contains only terms dependent on the number operator. Since they commute with the bosonic operators they have no influence on the system's dynamics but only on its chemical potential (see section VIIA).

\section{Diagonalization of the total Hamiltonian}

The total Hamiltonian in the forward scattering approximation,

$$
\begin{aligned}
H_{T}= & H_{0}+H_{I} \\
= & \hbar \omega_{c} \sum_{n>0} \sum_{m} n b_{n, m}^{\dagger} b_{n, m} \\
& +\frac{e^{2}}{\varepsilon l} \sum_{n>0} \sum_{m, m^{\prime}} g\left(n ; m, m^{\prime}\right) b_{n, m}^{\dagger} b_{n, m^{\prime}}+H_{N^{\prime}},
\end{aligned}
$$

with $g\left(n ; m, m^{\prime}\right)=g_{d i}\left(n ; m, m^{\prime}\right)+g_{e x}\left(n ; m, m^{\prime}\right)$, can be diagonalized by an appropriate linear combination of guiding center bosonic operators $b_{n, m}^{\dagger}$. It is easy to see that such a combination is given by

$$
b_{n}^{\dagger}(\bar{q})=\sum_{m=0}^{N_{\Phi}-1} \Phi_{m}(\bar{q}) b_{n, m}^{\dagger}, \quad \Phi_{m}(\bar{q})=\sqrt{\bar{q}} L_{m}\left(\frac{\bar{q}^{2}}{2}\right) e^{-\bar{q}^{2} / 4} .
$$

This new representation describes the degeneracy breaking between excitations like (a) and (d) in Fig.(1). By using the completeness and orthogonality relations of the $\Phi_{m}(q)$ functions,

$$
\begin{aligned}
\sum_{m=0}^{N_{\phi}-1} \Phi_{m}(\bar{q}) \Phi_{m}\left(\bar{q}^{\prime}\right) & =\delta\left(\bar{q}-\bar{q}^{\prime}\right) \\
\int_{0}^{\infty} d \bar{q} \Phi_{m}(\bar{q}) \Phi_{m^{\prime}}(\bar{q}) & =\delta_{m, m^{\prime}}
\end{aligned}
$$

we see that the operators $b_{n}(\bar{q})$ also obey bosonic commutations relations

$$
\left[b_{n}(\bar{q}), b_{n^{\prime}}^{\dagger}\left(\bar{q}^{\prime}\right)\right]=\delta_{n, n^{\prime}} \delta\left(\bar{q}-\bar{q}^{\prime}\right), \quad\left[b_{n}(\bar{q}), b_{n^{\prime}}\left(\bar{q}^{\prime}\right)\right]=0,
$$

and are related to the $b_{n, m}^{\dagger}$ bosons by

$$
b_{n, m}^{\dagger}=\int_{0}^{\infty} d \bar{q} \Phi_{m}(\bar{q}) b_{n}^{\dagger}(\bar{q}) .
$$

The same holds for the operator $\hat{N}(\bar{q})$, which plays the role of a "zero-mode boson":

$$
\hat{N}(\bar{q})=\sum_{m=0}^{N_{\phi}-1} \Phi_{m}(\bar{q}) \hat{N}_{m}
$$

The total Hamiltonian can then be directly rewritten in this new representation as

$$
H_{T}=\sum_{n>0} \int d \bar{q} E_{n}(\bar{q}) b_{n}^{\dagger}(\bar{q}) b_{n}(\bar{q})+H_{N^{\prime}},
$$

with the energy spectrum

$$
E_{n}(\bar{q})=n\left[\hbar \omega_{c}+\tilde{V}_{e x}^{n}(\bar{q})+\tilde{V}_{d i}^{n}(\bar{q})\right]+E_{\text {Self }}^{n} .
$$

The fact that the Hamiltonian is diagonal in the basis $b_{n}(\bar{q})$ is a consequence of the forward scattering approximation. In the symmetric gauge, the system's angular momentum is given by $L=\sum_{i}\left(n_{i}-m_{i}\right)$ and is a constant of motion. Therefore, in (2.6), the processes with non-zero matrix elements are the ones at which

$$
\left(n_{1}^{\prime}-m_{1}^{\prime}\right)+\left(n_{2}^{\prime}-m_{2}^{\prime}\right)=\left(n_{1}-m_{1}\right)+\left(n_{2}-m_{2}\right) .
$$

In the forward scattering approximation we have $m_{1}=m_{1}^{\prime}$ and $m_{2}=m_{2}^{\prime}$ (or $m_{1}=m_{2}^{\prime}$ and $m_{2}=m_{1}^{\prime}$, in the exchange processes). Eq.(3.18) is then reduced to $\Delta n_{1}+\Delta n_{2}=0$, with $\Delta n_{i}=n_{i}^{\prime}-n_{i}$. So, we are restricted to the first order terms in a degenerate perturbation theory, which present only terms that conserve the energy associated with the free portion, $H_{0}$, of the total Hamiltonian. In the bosonic representation this means terms like $b_{n}^{\dagger}(\bar{q}) b_{n}(\bar{q})$, but neither like $b_{n}^{\dagger}(\bar{q}) b_{n^{\prime}}(\bar{q})$, with $n \neq n^{\prime}$, nor like $b_{n}^{(\dagger)}(\bar{q}) b_{n^{\prime}}^{(\dagger)}(\bar{q})$. Formally this form appears in our expressions after the angular integration over $\arg (\mathbf{q})$ in the matrix elements (see Appendix B), which clearly imposes the angular momentum conservation. 

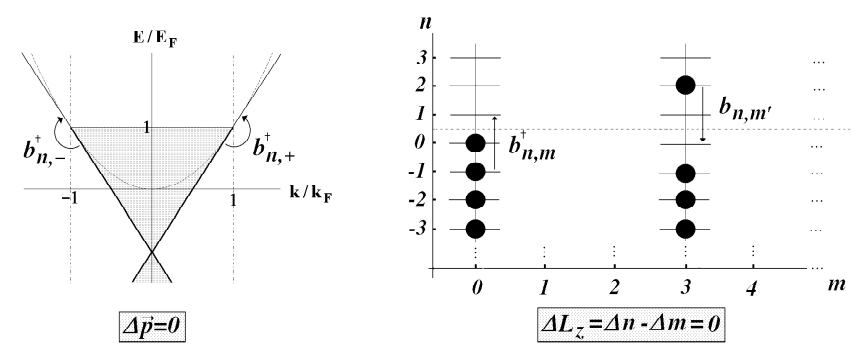

Figure 2: (a) Illustration of the linear momentum conservation by the action of $b_{n,+}^{\dagger} b_{n,-}^{\dagger}$ in the Tomonaga-Luttinger model. (b) Illustration of the angular momentum conservation by the action of the $b_{n, m}^{\dagger} b_{n, m^{\prime}}$ in our model. The particles are kept in their channels, therefore $\Delta m=$ 0 , and the variation on $n, \Delta n$, must be equal to zero too.

\section{COMPARISON WITH THE TOMONAGA-LUTTINGER MODEL AND THE 2D-BOSONIZATION}

We can compare our Hamiltonian (3.10) with the one of the Tomonaga-Luttinger model ${ }^{27,28}$ :

$$
\begin{aligned}
H= & \sum_{n>0} k_{n}\left\{\sum_{j= \pm}\left[\hbar v_{F}+g_{4}\left(k_{n}\right)\right] b_{n, j}^{\dagger} b_{n, j}\right. \\
& \left.+\sum_{j \neq j^{\prime}} g_{2}\left(k_{n}\right)\left(b_{n, j}^{\dagger} b_{n, j^{\prime}}^{\dagger}+b_{n, j} b_{n, j^{\prime}}\right)\right\}+H_{N},
\end{aligned}
$$

associated with one-dimensional (1D) fermion systems. In this model the system's neutral excitations are distributed in two momentum channels, $j= \pm$ (left and right-moving fermions), presenting energies given by the linearization of the dispersion relation close to the two Fermi points, $\pm k_{F}$ (see Fig.(2)). In our two dimensional model the neutral excitations are distributed among the $m=N_{\phi}$ guiding centers. Comparing (3.10) and (4.1) we see that, despite the different number of channels, we have the equivalence relations:

$$
g_{4}\left(k_{n}\right) \rightarrow g(n ; m, m), \quad g_{2}\left(k_{n}\right) \rightarrow g\left(n ; m, m^{\prime}\right) .
$$

In the Tomonaga-Luttinger model the terms associated with the interaction of particles in different channels, described by $g_{2}\left(k_{n}\right)$, present the form $b_{n, j}^{\dagger} b_{n, j^{\prime}}^{\dagger}$ or $b_{n, j} b_{n, j^{\prime}}$. This is because of the linear momentum conservation in the system: $b_{n,+}^{\dagger}$ creates an excitation with wave vector $k_{F}+k_{n}$, while $b_{n,-}^{\dagger}$ creates an excitation with wave vector $-k_{F}-k_{n}$. So, $b_{n, j}^{\dagger} b_{n, j^{\prime}}^{\dagger}$ creates an excitation with total momentum equal to zero (see Fig.(2)).

In the Tomonaga-Luttinger model this portion of the Hamiltonian, associated with $g_{2}\left(k_{n}\right)$, does not commute with the kinetic energy term $H_{0}=\hbar v_{F} \sum_{n>0} \sum_{j= \pm} k_{n} b_{n, j}^{\dagger} b_{n, j}$. Therefore, it modifies the system's ground state, which has a distribution of electron-hole pairs. The terms associated with $g_{4}\left(k_{n}\right)$ describe the interaction between particles in the same channel. These terms commute with $H_{0}$ and their influence is limited to removing degeneracies in the excitations ${ }^{27}$. In our model, all the terms associated with the interparticle interaction, in the same or different channels, present the same form $b_{n, m}^{\dagger} b_{n, m^{\prime}}$. As we have seen in the section above (after having made the forward scattering approximation) this form results from the angular momentum conservation, which does not allow the presence of terms like $b_{n, m}^{\dagger} b_{n, m^{\prime}}^{\dagger}$ or $b_{n, m} b_{n, m^{\prime}}$ (see Fig.(2)). Therefore, we will have a ground state like a Fermi sea, without particle-hole excitations or Landau levels mixing. All the interactions $g\left(n ; m, m^{\prime}\right)$, with $m=m^{\prime}$ or $m \neq m^{\prime}$, contribute to removing the degeneracies of the $n$ levels.

In a bosonized Hamiltonian, the presence of terms like $b^{\dagger} b^{\dagger}$ and $b b$, which separately do not conserve energy, "spreads" the single-particle spectral functions and gives rise to the anomalous dimension, $\alpha$, in the correlations functions ${ }^{37}$. In the spin-full Tomonaga-Luttinger model, besides the spincharge separation effect, the presence of this anomalous dimension results in the absence of fermionic quasi-particles (vanishing of the quasi-particle residue $z_{k} \sim\left|k-k_{F}\right|^{\alpha}$ as $k \rightarrow$ $\left.k_{F}\right)$. In other words in the non-Fermi liquid behavior of the model. In the $2 \mathrm{D}$ bosonization of the electron gas ${ }^{20}$ terms like $b^{\dagger} b^{\dagger}$ and $b b$, although present in the original interaction Hamiltonian, can be shown to vanish in the thermodynamic limit and in the asymptotic low-energy regime, giving rise to the Fermi liquid behavior. In our model it is clear that we will not have effects coming from an anomalous dimension, but we will have other effects that will give rise to a non-normal behavior.

\section{INCLUDING THE ELECTRON SPIN}

\section{A. The Free Hamiltonian}

Including the electron spin, the free Hamiltonian (2.5) becomes:

$$
H_{0}=\hbar \omega_{c} \sum_{s= \pm} \sum_{m=0}^{N_{\Phi}-1} \sum_{n=0}^{\infty}(n+1 / 2) c_{n, m, s}^{\dagger} c_{n, m, s} .
$$

Considering the extension of the Hilbert space to negative energy values, we can write $H_{0}$ in terms of the new bosonic operators, $b_{n, s}(\bar{q})$ :

$$
\begin{gathered}
H_{0}=\hbar \omega_{c} \sum_{s= \pm} \int d \bar{q}\left[\sum_{n>0} n b_{n, s}^{\dagger}(\bar{q}) b_{n, s}(\bar{q})+\frac{1}{2} \hat{N}_{s}^{2}(\bar{q})\right] \\
b_{n, s}(\bar{q})=\sum_{m=1}^{N_{\phi}-1} \Phi_{m}(\bar{q}) \frac{1}{\sqrt{n}} \sum_{p=-\infty}^{\infty} c_{p, m, s}^{\dagger} c_{p+n, m, s} .
\end{gathered}
$$

However, it is more convenient to define the following usual bosonic operators ${ }^{27}$ :

$$
\begin{aligned}
& b_{n}(\bar{q})_{\rho}=\frac{1}{\sqrt{2}}\left[b_{n,+}(\bar{q})+b_{n,-}(\bar{q})\right], \\
& b_{n}(\bar{q})_{\sigma}=\frac{1}{\sqrt{2}}\left[b_{n,+}(\bar{q})-b_{n,-}(\bar{q})\right],
\end{aligned}
$$

which are, as we will see, directly related to the charge and spin fluctuations in our system. Writing (5.2) in terms of these 
operators, we have:

$$
H_{0}=\hbar \omega_{c} \sum_{i=\rho, \sigma} \int d \bar{q}\left[\sum_{n>0} n b_{n}^{\dagger}(\bar{q})_{i} b_{n}(\bar{q})_{i}+\frac{1}{2} \hat{N}^{2}(\bar{q})_{i}\right] .
$$

The bosonic operators $b_{n}(q)_{\rho}$ and $b_{n}(q)_{\sigma}$ commute. Therefore, they are associated with two different set of excitations. In one dimensional systems they describe the independent charge and spin fluctuations $27,29,30$. In our system these operators play an equivalent role. If the higher Landau levels have both spin sublevels filled (see the next section) the system ground state will present total spin $\mathbf{S}=\mathbf{0}$. Therefore, we will have triplet or singlet excitations. The triplet excitations, described by the operators $b_{n}^{(\dagger)}(\bar{q})_{\rho}$, give rise to poles in the density response function $\chi_{\rho}(q, \omega)$ whereas the singlet ones, described by the operators $b_{n}^{(\dagger)}(\bar{q})_{\sigma}$, to poles in the spindensity response function $\chi_{\sigma}(q, \omega) 4$.

\section{B. The Zeeman Energy}

The inclusion of the electron spin brings together the Zeeman energy, $H_{Z e}=\frac{\hbar}{2} \mu_{B} g B \sum_{s= \pm} \sum_{n, m} s c_{n, m, s}^{\dagger} c_{n, m, s}$, which splits each Landau level into two sublevels with energy separation of the order of $\Delta E_{z}=\frac{\hbar}{2} \mu_{B} g B$. However, we will treat only excitations between Landau levels. Our bosonic operators $b_{n}(\bar{q})_{\rho}$ or $b_{n}(\bar{q})_{\sigma}$ are associated with particle-hole pairs with the same spin, see eqs. (5.3 5.5). These are the only possible excitations for a system with both spin sublevels filled $(\mathbf{S}=0)$. The particle's spin will be conserved in the transitions and therefore the Zeeman energy will not have any influence in the system dynamics. One can easily see that $H_{Z e}$ commutes with $b_{n}(\bar{q})_{\rho}$ and $b_{n}(\bar{q})_{\sigma}$. An appropriate description of the intra-Landau levels excitations ${ }^{31}$, through an appropriate bosonization method, was also presented recently by one of us 32 .

\section{The Interacting Hamiltonian}

We saw that the inclusion of the spin and the definition of the operators (5.4 5.5) bring the free Hamiltonian $H_{0}$ (5.1) to the form (5.6). Now, once the exchange processes occur only between particles with the same spin, the exchange Hamiltonian $H_{I}^{E x}$, (B3), will be diagonal in the $s$ index, like $H_{0}$, (5.1). Therefore, it will be transformed in the same way as $H_{0}$, being written in terms of $b_{n}(\bar{q})_{\rho}$ and $b_{n}(\bar{q})_{\sigma}$. On the other hand, the direct interaction $H_{I}^{D i r},(\underline{\mathrm{B} 2})$, will not be diagonal in the spin index. The sum over the spin indices, $\sum_{s, s^{\prime}}$, results in the cancellation of the terms proportional to $b_{n}(\bar{q})_{\sigma}$ and in the presence of an interaction equal to $2 \tilde{V}_{d i}^{n}(\bar{q})$ associated with the charge operators $b_{n}(\bar{q})_{\rho}$. Physically, the direct interaction gives rise only to charge excitations, while the exchange interaction gives rise to charge and spin excitations. The final Hamiltonian will then present the charge and spin fluctuations separated:

$$
\begin{aligned}
H_{T} & =H^{(\rho)}+H^{(\sigma)} \\
H^{(i)} & =\sum_{n>0} \int d \bar{q} E_{n}^{(i)}(\bar{q}) b_{n}^{\dagger}(\bar{q})_{i} b_{n}(\bar{q})_{i}, \\
E_{n}^{(i)}(\bar{q}) & =n\left[\hbar \omega_{c}+2 \delta_{i, \rho} \tilde{V}_{d i}^{n}(\bar{q})+\tilde{V}_{e x}^{n}(\bar{q})\right]+E_{\text {Self }}^{n},
\end{aligned}
$$

with $i=\rho, \sigma$ and the terms of the energy spectrum $E_{n}^{(i)}(\bar{q})$ given in 3.7 3.9). We did not write the terms proportional to the number operators. In the limit $q \rightarrow \infty$ we will have the asymptotic value $E_{n}^{(i)}=n \hbar \omega_{c}+E_{\text {Self }}^{n}$ since $\tilde{V}_{e x}^{n}(\bar{q})$ and $\tilde{V}_{d i}^{n}(\bar{q})$ goes to zero.

\section{SPECTRUM OF NEUTRAL EXCITATIONS}

For the first excited level, $n=1$, the neutral excitation spectrum that we have obtained, eq. [5.8), is identical to the one determined in eq.(4.2) of the Kallin and Halperin's paper ${ }^{4}$. We just have to perform the angular integration over $\arg (\mathbf{r})$ in the latter to obtain (5.8). For $n>1$ the only difference is the $n$ factor multiplying $\tilde{V}_{d i}^{n}(\bar{q})$ and $\tilde{V}_{e x}^{n}(\bar{q})$, that we will discuss below. The authors of Ref.[4] clarify that (5.8), within the strong field approximation, applies for two cases, at which it is exact to first order in $\left(e^{2} / \varepsilon l\right) / \hbar \omega_{c}$ : (i) excitations associated with $n=1$ in systems with arbitrary $\mathrm{v}$ and (ii) excitations associated with arbitrary $n$ since the system presents $v=1$; in both cases the system must present the two spin Landau sublevels filled, as we suppose in sec.( $(\mathrm{VB})$. Despite these restrictions, these are the excitations of major interest. Outside the domain of these conditions eq.(5.8) represents a less controlled approximation, once the multi-exciton states were neglected.

In the bosonization procedure we can easily understand the origin of these restrictions. To obtain a precise bosonic description of the excitations we have approximated all the matrix elements $G_{p, p+n}$, that describe the excitation of an electron to the $p+n$ level, leaving a hole in the $p$ level, by the Fermi level matrix element, $\left.G_{p, p+n}\right|_{p=v-1}=G_{v-1, v-1+n}$, see Appendix B. In this case we have a precise description for the excitations associated with $n=1$, as they are represented by the unique element $G_{v-1, v}$, and for the arbitrary excitations in a system presenting $\mathrm{v}=1$, since they are all associated with the same element $G_{0, n}$. In these two cases all the particle-hole pairs that contribute to a given excitation are associated with the same Landau levels. So, we do not have to consider all the $n$ Landau levels between $v-n$ and $v-1$ that would contribute to a given $E_{n}(q)$ if $v-n>0^{4}$. However, once we introduce negative energy states, even for filling $v=1$ we will have a contribution of $n$ states. This is the origin of the $n$ factor multiplying $\tilde{V}_{d i}^{n}(\bar{q})$ and $\tilde{V}_{e x}^{n}(\bar{q})$ in (5.8). But, as we will see, the $n=1$ magnetoplasma mode will be the most import excitation in our analysis and, in the strong field limit $v \hbar \omega_{c} \gg e^{2} / \varepsilon l$, the higher magnetoplasmon modes will not present a relevant modification due this $n$ factor.

We justify the approximation in the matrix elements, $\left.G_{p, p+n}\right|_{p=v-1}=G_{v-1, v-1+n}$, with the strong field approximation, see Appendix B. However, we should mention that it is 
also a good approximation to describe the low energy excitations $(n \ll v)$ of a system in low magnetic field $(v \gg 1)$. In this case, for small wave vectors $\bar{q}$, it can be shown that the matrix elements $G_{v-1, v-1+n}(\bar{q})$ depend approximately only on $n$ and the value adopted by $\mathrm{v}$ is irrelevant. This property was explored in a similar bosonization method ${ }^{16}$.

The dispersion curves for the modes close to $\hbar \omega_{c}$ are obtained calculating (5.8) for $n=1$. For $v=1^{33}$, e.g., we got:

$$
\begin{aligned}
E_{\mathrm{v}=n=1}^{(i)}(\bar{q})= & \hbar \omega_{c}+\frac{e^{2}}{\varepsilon l} \frac{1}{2} \sqrt{\frac{\pi}{2}}\left\{1-e^{-\frac{\bar{q}^{2}}{4}}\left[\left(1+\frac{\bar{q}^{2}}{2}\right) I_{0}\left(\frac{\bar{q}^{2}}{4}\right)\right.\right. \\
& \left.\left.-\frac{\bar{q}^{2}}{2} I_{1}\left(\frac{\bar{q}^{2}}{4}\right)\right]+2 \delta_{i, \rho} \sqrt{\frac{2}{\pi}} \bar{q} e^{-\frac{\bar{q}^{2}}{2}}\right\}
\end{aligned}
$$

These expressions, for the charge and spin modes, $i=\rho, \sigma$, are exactly the eqs. (4.4) and (4.8) in Ref.[4] or (3.3) in Ref.[31]. The first term between curly brackets is independent of $q$ and corresponds to $E_{\text {Self }}^{n=1}$. The second term corresponds to $\tilde{V}_{e x}^{n=1}(\bar{q})$ and the last one to $2 \delta_{i, \rho} \tilde{V}_{d i}^{n=1}(\bar{q})$. For higher $n$ or $v$ values we have much longer expressions, so we do not explicitly show them here.

\section{SINGLE-PARTICLE PROPERTIES}

\section{A. Fermionic Operator}

The bosonic representation of fermionic fields, also known as the Mattis-Mandelstam formula, is an identity in one dimensional systems if the theory can be formulated in terms of a set of fermionic operators, $\left\{c_{k, j}, c_{k^{\prime}, j^{\prime}}^{\dagger}\right\}=\delta_{j, j^{\prime}} \delta_{k, k^{\prime}}$, which are labeled by the "species" index $j=1, \ldots, N_{j}$ and a discrete and unbounded "momentum" index $k$, which typically labels the eingenenergies, $E_{k}$, of the non-interacting system ${ }^{18}$. The fermionic fields, $\psi_{j}(x)$, are defined as:

$$
\psi_{j}(x) \equiv \sqrt{\frac{2 \pi}{L}} \sum_{n=-\infty}^{\infty} e^{-i k_{n} x} c_{n, j}
$$

where $L$ is the length associated with the system size and $c_{n, j}$ remove the fermion from the state $k_{n}=\frac{2 \pi}{L} n$. They have the following bosonic representation ${ }^{18,35}$ :

$$
\begin{aligned}
\psi_{j}(x) & =\frac{1}{\sqrt{\varepsilon}} U_{j} e^{-i \frac{2 \pi}{L} \hat{N}_{j} x} e^{-i \Phi_{j}(x)} \\
\Phi_{j}(x) & =i \sum_{k_{n}>0} \frac{e^{-\varepsilon k_{n} / 2}}{\sqrt{n}}\left(e^{-i k_{n} x} b_{n, j}-e^{i k_{n} x} b_{n, j}^{\dagger}\right) \\
b_{n, j} & =\frac{1}{\sqrt{n}} \sum_{p=-\infty}^{\infty} c_{p-n, j}^{\dagger} c_{p, j}
\end{aligned}
$$

where $b_{n, j}$ are bosonic operators 18 and $\varepsilon>0$ is an infinitesimal regularization parameter such that $\varepsilon \rightarrow 0^{+}$at the end of the calculations.
We get a fermionic field appropriate for a bosonic representation with $b_{n, m}$, introducing the phase representation ${ }^{16}$

$$
\psi_{m}(\theta) \equiv \frac{1}{\sqrt{2 \pi}} \sum_{n=-\infty}^{\infty} e^{-i n \theta} c_{n, m}
$$

In this way the set of operators $\left\{c_{n, m}, b_{n, m}, \hat{N}_{m}, \psi_{m}(\theta)\right\}$ is completely analogous to the set $\left\{c_{k_{n}, j}, b_{n, j}, \hat{N}_{j}, \psi_{j}(x)\right\}$ if we make the replacements: $j \rightarrow m, x \rightarrow \theta, k_{n} \rightarrow n, \frac{2 \pi}{L} \hat{N}_{j} \rightarrow \hat{N}_{m}$ and $\sqrt{\frac{2 \pi}{L}} \rightarrow \frac{1}{\sqrt{2 \pi}}$. Therefore, writing the fermionic field $\psi_{m}(\theta)$ in terms of the operators $b_{n}(\bar{q})$ of (3.11), it presents the following representation, analogous to (7.2.7.4):

$$
\begin{aligned}
\psi_{m}(\theta)= & \lim _{\varepsilon \rightarrow 0} \frac{1}{\sqrt{2 \pi \varepsilon}} U_{m} e^{-i \hat{N}_{m} \theta} e^{-i \Theta_{m}(\theta)} \\
\Theta_{m}(\theta)= & i \int_{0}^{\infty} d \bar{q} \Phi_{m}(\bar{q}) \sum_{n=1}^{\infty} \frac{e^{-\frac{n \varepsilon}{2}}}{\sqrt{n}}\left[e^{-i n \theta} b_{n}(\bar{q})\right. \\
& \left.-e^{i n \theta} b_{n}^{\dagger}(\bar{q})\right]
\end{aligned}
$$

In order to determine the time evolution of the fermion field (7.6) we first note that $b_{n}(\bar{q}, t)=e^{-i E_{n}(\bar{q}) t} b_{n}(\bar{q})$, with $E_{n}(\bar{q})$ given by (3.17), and that the time evolution of $U_{m}$ is given by

$$
U_{m}(t)=e^{i H_{N}^{m} t} U_{m} e^{-i H_{N}^{m} t}=e^{i\left(H_{N}^{m}-\left.H_{N}^{m}\right|_{N_{m}+1}\right) t} U_{m}
$$

where $H_{N}^{m}$ is the portion of the total Hamiltonian proportional to the $\hat{N}_{m}$ operator. Acting on the ground state we get $\langle 0| U_{m}(t)=e^{-i \mu_{m}^{+} t}\langle 0| U_{m}$, where $\mu_{m}^{+}$corresponds to the chemical potential associated with the creation of a particle in the $m$ th channel, $\mu_{m}^{+}=E_{0}\left(N_{m}+1\right)-E_{0}\left(N_{m}\right)$. Grouping the terms proportional to $N$ in (3.10), which are given in the Appendix $\mathrm{B}$, we get

$$
\mu_{m}^{+}=\hbar \omega_{c}-\frac{1}{2} V(r=0)+\frac{1}{2 N_{\Phi}} \frac{\tilde{V}(q=0)}{2 \pi l^{2}}=\mu^{+}
$$

\section{B. Retarded Green's Functions at $T=0$}

The retarded Green's function, at temperature $T=0$, associated with a particle in the quantum state $|n, m\rangle$ (with $n$ measured from the Fermi level, see Fig.(1) is given by ${ }^{25}$ :

$$
\begin{aligned}
i G_{n, m}^{R e t}(t) & =\theta(t)\left\langle c_{n, m}(t) c_{n, m}^{\dagger}(0)+c_{n, m}^{\dagger}(0) c_{n, m}(t)\right\rangle_{0} \\
& =i \theta(t) \int_{0}^{2 \pi} d \theta e^{i n \theta}\left[G_{m, \theta}^{>}(t)+G_{m, \theta}^{<}(t)\right],(7 . \\
i G_{m, \theta}^{>}(t) & =\left\langle\psi_{m}(\theta, t) \psi_{m}^{\dagger}(0,0)\right\rangle_{0}, \\
i G_{m, \theta}^{<}(t) & =\left\langle\psi_{m}^{\dagger}(0,0) \psi_{m}(\theta, t)\right\rangle_{0},
\end{aligned}
$$

where we use the relation 7.5 between the fields $\psi_{m}(\theta)$ and $c_{n, m}$. The calculation of $G_{m, \theta}^{\gtrless}(t)$, from the representation 
(7.6), is presented in Appendix C. We get:

$$
\begin{aligned}
i G_{n, m}^{R e t}(t)= & \theta(t) \int_{0}^{2 \pi} d \theta e^{i n \theta}\left[e^{-i \mu^{+} t} e^{-i \theta} K_{m, \theta}(t)\right. \\
& \left.+e^{-i \mu^{-} t} K_{m, \theta}^{*}(t)\right] \\
K_{m, \theta}(t)= & \frac{1}{2 \pi} e^{\sum_{n=1}^{\infty} \frac{e^{-n \varepsilon}}{n} e^{-i n \theta} \int_{0}^{\infty} d \bar{q} \Phi_{m}^{2}(\bar{q}) e^{-i E_{n}(\bar{q}) t}}
\end{aligned}
$$

where $\mu^{-}$corresponds to the chemical potential associated with the removal of a system's particle.

We can calculate the retarded Green's function associated with different states. For a particle in a position $\mathbf{r}$ we have

$$
\begin{aligned}
i G^{R e t}(\mathbf{r}, t) & =\theta(t)\left\langle\psi(\mathbf{r}, t) \psi^{\dagger}(\mathbf{r}, 0)+\psi^{\dagger}(\mathbf{r}, 0) \psi(\mathbf{r}, t)\right\rangle \\
& =i \sum_{n^{\prime}=0}^{\infty} \sum_{m=0}^{N_{\phi}-1}\left|\left\langle\mathbf{r} \mid n^{\prime}, m\right\rangle\right|^{2} \tilde{G}_{n^{\prime}, m}^{R e t}(t) .
\end{aligned}
$$

where we use the representation of $\psi(\mathbf{r})$ in the Landau level basis, (2.2), and $\left\langle\mathbf{r} \mid n^{\prime}, m\right\rangle$ is given by (2.3). Notice that we defined $G_{n, m}^{\text {Ret }}(t)$, eq. (7.11), with the index $n$ relative to the Fermi level, according to Figs.(1) and (3), whereas the index $n^{\prime}$ above is relative to the single particle ground state. So we must write $n^{\prime}=n-(v-1)$ in $\tilde{G}_{n^{\prime}, m}^{R e t}(t)$, getting

$$
G^{R e t}(\mathbf{r}, t)=\sum_{n=0}^{\infty} \sum_{m=0}^{N_{\phi}-1}|\langle\mathbf{r} \mid n, m\rangle|^{2} G_{n-(v-1), m}^{R e t}(t) .
$$

If we want the Green's function associated with a particle in a different state, e.g., the wave vector $\mathbf{q}$, we have to replace $\langle\mathbf{r} \mid n, m\rangle$ by $\langle\mathbf{q} \mid n, m\rangle$.

\section{Single-Particle Spectral Functions}

Now we will determine the single-particle spectral functions, $A(\omega)$, which gives the spectral distribution generated by the inclusion or removal of a particle in the system. The spectral function associated with a particle in the sate $|n, m\rangle$ is given by

$$
A_{n, m}(\omega)=-\frac{1}{\pi} \operatorname{Im} G_{n, m}^{R e t}(\omega)=-\frac{1}{\pi} \operatorname{Im} \int_{-\infty}^{\infty} d t e^{-i \omega t} G_{n, m}^{R e t}(t) .
$$

We need the Fourier transform of the function $G_{n, m}^{\text {Ret }}(t)$. We then take $n>0$, creating a particle or a hole above the Fermi level (see Fig.(1)). Since our ground state does not present electrons above the Fermi level, see section (IV), only the first term between square brackets of (7.11) will contribute to $A_{n>0, m}(\omega)$, describing the creation of an electron in the system. Substituting the expressions (7.11) and (7.12) in (7.15), we get

$$
\begin{aligned}
i G_{n>0, m}^{R e t}(t)= & \theta(t) \frac{1}{2 \pi} \int_{0}^{2 \pi} d \theta e^{i(n-1) \theta} e^{-i \mu^{+} t} \\
& \times e^{\sum_{\bar{n}=1}^{\infty} \frac{e^{-i \bar{n}}}{\bar{n}} \int_{0}^{\infty} d \bar{q} \Phi_{m}^{2}(\bar{q}) e^{-i E_{\bar{n}}(\bar{q}) t} .}
\end{aligned}
$$

Now, the exponential function of the sum $\sum_{\bar{n}=1}^{\infty}$ can be expanded in a series that, after the angular integration over $\theta$, will result in a finite number of terms for each $n$. It is easy to see that

$$
\begin{aligned}
i G_{n=1, m}^{R e t}(t)= & \theta(t) e^{-i \mu^{+} t} \\
i G_{n=2, m}^{R e t}(t)= & \theta(t) e^{-i \mu^{+} t} \int_{0}^{\infty} d \bar{q} \Phi_{m}^{2}(\bar{q}) e^{-i E_{1}(\bar{q}) t} \\
i G_{n=3, m}^{R e t}(t)= & \frac{1}{2} \theta(t) e^{-i \mu^{+} t} \int_{0}^{\infty} d \bar{q} \Phi_{m}^{2}(\bar{q})\left[e^{-i E_{2}(\bar{q}) t}\right. \\
& \left.+\int_{0}^{\infty} d \bar{q}^{\prime} \Phi_{m}^{2}\left(\bar{q}^{\prime}\right) e^{-i\left[E_{1}(\bar{q})+E_{1}\left(\bar{q}^{\prime}\right)\right] t}\right]
\end{aligned}
$$

and so on for $n>3$. Therefore, we see that for each $i G_{n, m}^{R e t}(t)$ we only have the contribution of the normal modes presenting $\bar{n}<n$. This behavior is general, occurring in any bosonized system that presents a discrete levels structure. Recently, it has also been observed in the treatment of the Luttinger liquid in a finite one-dimensional wire ${ }^{36}$, where the discrete levels structure came from the box-like boundary conditions. We can also see that we do not have the contribution of the mode $\bar{n}=n$. Therefore, according to (7.15) and (7.16), the spectral function associated with the creation of a particle in the first excited state, $n=1$, is given simply by

$$
A_{n=1, m}(\omega)=\delta\left(\omega-\mu^{+}\right) .
$$

In this situation the only energy level excited by the introduced particle is the one given by the chemical potential, without any contribution from the excitation of bosonic modes. This was expected as a consequence of the first order approximation. Consider the inclusion of a particle in a state $|n=1, m\rangle$, as presented in Fig.(3)a). Again, since our ground state has no particle-hole pairs, there is no empty state to which this particle could relax. Therefore, it cannot excite (or decay into) any of the bosonic modes of the system. The particle can neither move between the others channels, once the number of particles in each channel must be conserved. These means that the particle can not "see" the degeneracy breaking in $m$, associated with the dispersion $\Delta E_{n=1}(\mathbf{q})$ in the level it is included. It is easy to see that this is as a consequence of the first order perturbation theory. Due to the angular momentum conservation, the particle could jump to a channel $m^{\prime} \neq m$, e.g. $m^{\prime}=m+1$, only exciting another particle in another level $n$ above it, as presented in Fig.(44). However, this should be a virtual transition, once it does not conserve energy.

According (7.15) and (7.17), the other spectral functions will be given by:

$$
\begin{aligned}
A_{n=2, m}(\omega)= & \int_{0}^{\infty} d \bar{q} \Phi_{m}^{2}(\bar{q}) \delta\left(\omega-E_{1}(\bar{q})-\mu^{+}\right) \\
A_{n=3, m}(\omega)= & \frac{1}{2} \int_{0}^{\infty} d \bar{q} \Phi_{m}^{2}(\bar{q})\left\{\left[\delta\left(\omega-E_{2}(\bar{q})-\mu^{+}\right)+\quad(7.19)\right.\right. \\
& \left.\left.\int_{0}^{\infty} d \bar{q}^{\prime} \Phi_{m}^{2}\left(\bar{q}^{\prime}\right) \delta\left(\omega-E_{1}(\bar{q})-E_{1}\left(\bar{q}^{\prime}\right)-\mu^{+}\right)\right]\right\}
\end{aligned}
$$

and so on. It is clear, from the scheme of Fig.(3), that the above expressions correspond to the distribution (or decay) of 


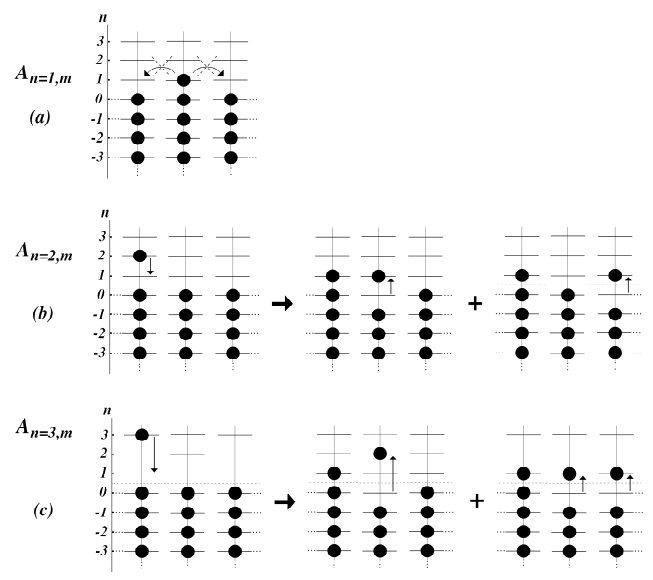

Figure 3: Scheme representing the distribution, in the bosonic modes, of the energy of the particle introduced in the system: (a) a particle created in the $n=1$ level can not excite any bosonic mode, (b) created in the level $n=2$ can excite the bosonic modes associated with $n=1, E_{n=1}(\bar{q})$, (c) created in the $n=3$ can excite the bosonic modes associated with $n=1, E_{n=1}(\bar{q})$, and $n=2, E_{n=2}(\bar{q})$, and so on.
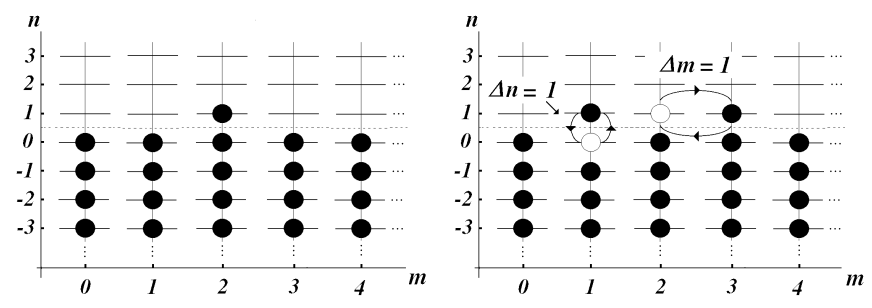

Figure 4: Scheme representing the "difficulty" (impossibility in first order) of a particle added to the system in the first excited level (a) to make a transition to the neighbor channel, with $\Delta m=+1$ (b). To conserve angular momentum, $\Delta L_{z}=\Delta n-\Delta m=0$, we must have another particle making a transition $\Delta n=+1$ simultaneously. This must be a virtual transition, once it does not conserve the energy.

a single-particle excitation in a set of collective bosonic excitations of total energy close to the free energy of the particle introduced. As the energy of the introduced particle increases it excites a larger number of collective modes. This kind of distribution is the essence of the bosonization methods ${ }^{37}$. In the next section, after we include the particle spin, we will present the graphic representation of some of this spectral functions (see Fig.(5)).

Analyzing the above expansion of $G_{n, m}^{R e t}(t)$ and recalling that $\int_{0}^{\infty} d \bar{q} \Phi_{m}^{2}(\bar{q})=1$ we see that $A_{n, m}(\omega)$ obeys the sum rule $\int_{-\infty}^{\infty} d \omega A_{n, m}(\omega)=1$, for all $\{n, m\}$.

The spectral density associated with the inclusion of a particle on the position $\mathbf{r}$ is given by

$A(\mathbf{r}, \omega)=-\frac{1}{\pi} \operatorname{Im} G^{R e t}(\mathbf{r}, \omega)=\sum_{n=0}^{\infty} \sum_{m=0}^{N_{\phi}-1}|\langle\mathbf{r} \mid n, m\rangle|^{2} A_{n-(v-1), m}(\omega)$,

where we use the relation (7.14) between $G^{R e t}(\mathbf{r}, t)$ and $G_{n-(v-1), m}^{R e t}(t)$. We verify that $\int_{-\infty}^{\infty} d \omega A(\mathbf{r}, \omega)=1$, once we have the correct normalization $\sum_{n, m}|\langle\mathbf{r} \mid n, m\rangle|^{2}=1$. Particularly, for the creation of a particle at the origin, $\mathbf{r}=\mathbf{0}$, we have

$$
A(\mathbf{r}=\mathbf{0}, \omega)=\frac{1}{(2 \pi l)^{2}} \sum_{n=0}^{\infty} A_{n-(v-1), n}(\omega)
$$

because $\langle\mathbf{0} \mid n, m\rangle=\delta_{n, m} / \sqrt{2 \pi l^{2}}$. One particle created at $\mathbf{r}=\mathbf{0}$ add to the system an angular momentum $\Delta L_{z}=0$. Therefore, it must decay only into the modes with $n=m$, which also present zero angular momentum and are indeed the only terms left in (7.20). We will see the graphic representation of $A(\mathbf{r}=\mathbf{0}, \omega)$ in Fig. (9), after the inclusion of the spin degree of freedom.

\section{Including the Electron Spin}

The details of the introduction of the electron spin, $s= \pm 1$, are presented in the Appendix D. For the Green's function we get

$$
\begin{aligned}
i G_{n, m, S}^{R e t}(t)= & \theta(t) \int_{0}^{2 \pi} d \theta e^{i n \theta}\left[e^{-i \mu_{s}^{+} t} e^{-i \theta} M_{m, \theta}(t)\right. \\
& \left.+e^{-i \mu_{s}^{-} t} M_{m, \theta}^{*}(t)\right] \\
M_{m, \theta}(t)= & \frac{1}{2 \pi} e^{\frac{1}{2} \sum_{n=1}^{\infty} \frac{1}{n} e^{-i n \theta} \int_{0}^{\infty} d \bar{q} \Phi_{m}^{2}(\bar{q})\left(e^{-i E_{n}^{(\rho)}(\bar{q}) t}+e^{-i E_{n}^{(\sigma)}(\bar{q}) t}\right),}
\end{aligned}
$$

with $E_{n}^{(i)}$ given by $(5.8)$.

The spectral function $A_{n, m, s}(\omega)$ presents the same structure of the equations (7.18) 7.19) under the following substitutions:

$$
\begin{aligned}
\delta\left(\omega-E_{n}(\bar{q})-\mu^{+}\right) & \rightarrow \frac{1}{2} \sum_{i=\rho, \sigma} \delta\left(\omega-E_{n}^{(i)}(\bar{q})-\mu_{s}^{+}\right), \\
\delta\left(\omega-E_{n}(\bar{q})-E_{n}\left(\bar{q}^{\prime}\right)-\mu^{+}\right) & \rightarrow \frac{1}{4}\left[\sum_{i=\rho, \sigma} \delta\left(\omega-E_{n}^{(i)}(\bar{q})-E_{n}^{(i)}\left(\bar{q}^{\prime}\right)-\mu_{s}^{+}\right)+2 \delta\left(\omega-E_{n}^{(\rho)}(\bar{q})-E_{n}^{(\sigma)}\left(\bar{q}^{\prime}\right)-\mu_{s}^{+}\right)\right],
\end{aligned}
$$

and so on. The functions $A_{n, m, s}(\omega)$ also obey the sum rule $\int_{-\infty}^{\infty} d \omega A_{n, m, s}(\omega)=1$, for all $\{n, m, s\}$.

In Fig. (5) we present examples of the form acquired by these spectral functions. The spectral function $A_{n=1, m, s}(\omega)$ corresponds simply to a $\delta$-function centered at the chemical potential energy $\mu_{s}^{+}$. From $A_{n=2, m, s}(\omega)$ on to higher values of $n$ we start to have a 
complex energy distribution. The particle excites simultaneously, according the scheme of Fig. (3. b), the charge and spin modes, associated with $E_{n=1}^{(\rho)}(\bar{q})$ and $E_{n=1}^{(\sigma)}(\bar{q})$. The spectral function $A_{n=2, m, s}(\omega)$ (see (7.19) can also be written as:

$$
A_{n=2, m, s}(\omega)=\frac{1}{2} \sum_{i=\rho, \sigma} \int_{0}^{\infty} \frac{d E(\bar{q})}{\left|\frac{d}{d \bar{q}} E(\bar{q})\right|} \Phi_{m}^{2}(\bar{q}) \delta\left(\omega-E_{n=1}^{(i)}(\bar{q})-\mu_{s}^{+}\right) \equiv \frac{1}{2} \sum_{i=\rho, \sigma_{\bar{q} \leftrightarrow E_{n=1}^{(i)}(\bar{q})+\mu_{s}^{+}=\omega}} \frac{\Phi_{m}^{2}(\bar{q})}{\left|\frac{d}{d \bar{q}} E_{n=1}^{(i)}(\bar{q})\right|}
$$

where we have performed the integration $\int_{0}^{\infty} d \bar{q}=\int_{0}^{\infty} \frac{d E(\bar{q})}{\left|\frac{d}{d \bar{q}} E(\bar{q})\right|}$ and $\sum_{\bar{q} \leftrightarrow E_{n=1}^{(i)}(\bar{q})+\mu_{s}^{+}=\omega}$ means a infinite sum over all the $\bar{q}$ values such that $E_{n=1}^{(i)}(\bar{q})+\mu_{s}^{+}$corresponds to the given $\omega$. So $A_{n=2, m, s}(\omega)$ will present peaks (integrable divergences), with weights proportional to $\Phi_{m}^{2}(\bar{q}) / 2$ where $d E_{n=1}^{(i)}(\bar{q}) / d \bar{q}=0$ and the system presents maxima in its density of states. This behavior is similar to the behavior of the dynamical structure factor, $S(q, \omega)$, measured by inelastic light-scattering ${ }^{7,10}$ in integer quantum Hall system, which confirm the existence of the rotons' minima in the dispersion of the collective modes. We can easily calculate $S(q, \omega)$ within the bosonization procedure, but since we obtain the same results presented in ${ }^{7}$ we do not present the calculation here.

The expression (7.24) is useful to understand the behavior of $A_{n, m, s}(\omega)$. However, the more direct way to evaluate it is substituting $\delta\left(\omega-E_{n=1}^{(i)}(\bar{q})-\mu_{s}^{+}\right)$by a Lorentzian of negligible width. This procedure allows us to compare the weight of the different peaks, while keeping the area under each one of them constant. In Fig. (6) we present the behavior of $A_{n=2, m=0, s}(\omega)$ for two different filling factors $v$. We see peaks in the energies of the extreme points of the charge and spin fluctuation modes and the energy of the particle being distributed between the minimum of $E_{n=1}^{(\sigma)}(\bar{q})$ and the maximum of $E_{n=1}^{(\rho)}(\bar{q})$. This behavior is analogous to the distribution of energy of an added particle in the Tomonaga-Luttinger model 137 . There the energy of a particle with momentum $q$ is partially distributed between the charge and spin modes, presenting dispersions $v_{\rho} q$ and $v_{\sigma} q$, and also over energies above and below these ones, due to the existence of the anomalous dimension. Here we do not have any effect of an anomalous dimension, see sec. (IV), however, both dispersion relations, $E_{n}^{(\rho)}(\bar{q})$ and $E_{n}^{(\sigma)}(\bar{q})$, present a non-monotonic behavior, giving rise to the many peaks in the spectral function. We also have the presence of the function $\Phi_{m}^{2}(\bar{q})$, which distributes the energy according to the relation between an excitation in the guiding center $m$ and one with wave vector $\bar{q}$, which we will discuss in sec VIIF

\section{E. Non-normal System}

In a non-interacting system, for $n \geq 1$, we would have $(\hbar=1)$

$$
A_{n, m, s}^{0}(\omega)=\delta\left(\omega-(n-1) \omega_{c}-\mu_{0, s}^{+}\right)
$$

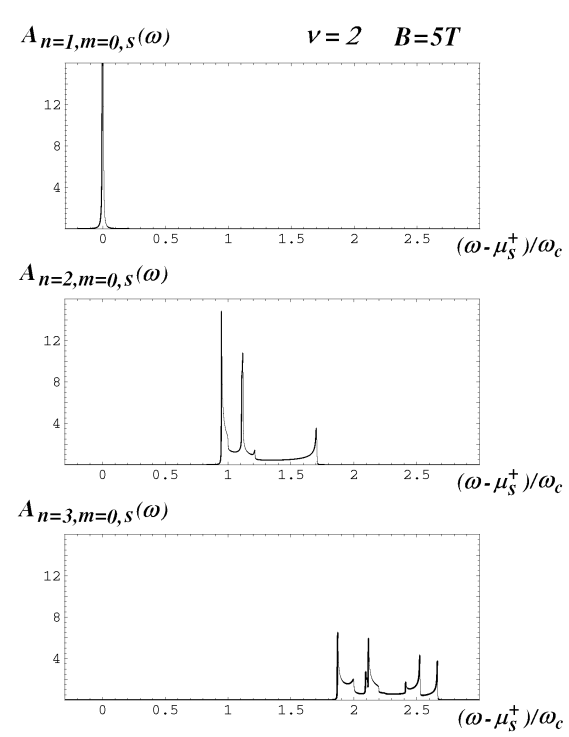

Figure 5: Examples of spectral functions $A_{n, m=0, s}(\omega)$ for a system with filling factor $v=2$ and a typical transverse magnetic field of $5 T$ in a GaAs heterojunction, where we have $\hbar \omega_{c}[K] \simeq 20 B[T]$ and $e^{2} / \varepsilon l[K] \simeq 50 \sqrt{B[T]}$. The $\delta$-functions in (7.23) where substituted by a Lorentzians of width $10^{-3} \omega_{c}$.

where $\mu_{0, s}^{+}=\hbar \omega_{c}+s \Delta E_{z}, \Delta E_{z}$ is the Zeeman energy, and the retarded Green's function would be given by

$$
G_{n, m, s}^{0, R e t}(\omega)=\frac{1}{\omega-(n-1) \omega_{c}-\mu_{0, s}^{+}+i \eta} .
$$

Now let us study what happens in the interacting system. For $n=1$, the introduction of the interaction results in a simple renormalization of the chemical potential $\mu_{0, s}^{+}$to $\mu_{s}^{+}$, similar to (7.9). For $n>1$ we have the spectral functions with the (integrable) divergences of Figs. (5) and (6). If we take the Fourier transform of $G_{n=2, m, s}^{R e t}(t)$, given by (7.22), we get

$$
G_{n=2, m, s}^{R e t}(\omega)=\frac{1}{2} \sum_{i=\rho, \sigma} \int_{0}^{\infty} d \bar{q} \frac{\Phi_{m}^{2}(\bar{q})}{\omega-E_{n=1}^{(i)}(\bar{q})-\mu_{s}^{+}+i \eta} .
$$

So, instead of a single pole, as in (7.25), we have a continuum of poles which give rise to some integrable divergences in the spectral functions. Therefore, we see that the effect of the interaction on the non-interacting spectral function is very different from shifting and broadening the non-interacting peak. It generates peaks with different energies and weights, associated with both charge and spin fluctuations. In normal 

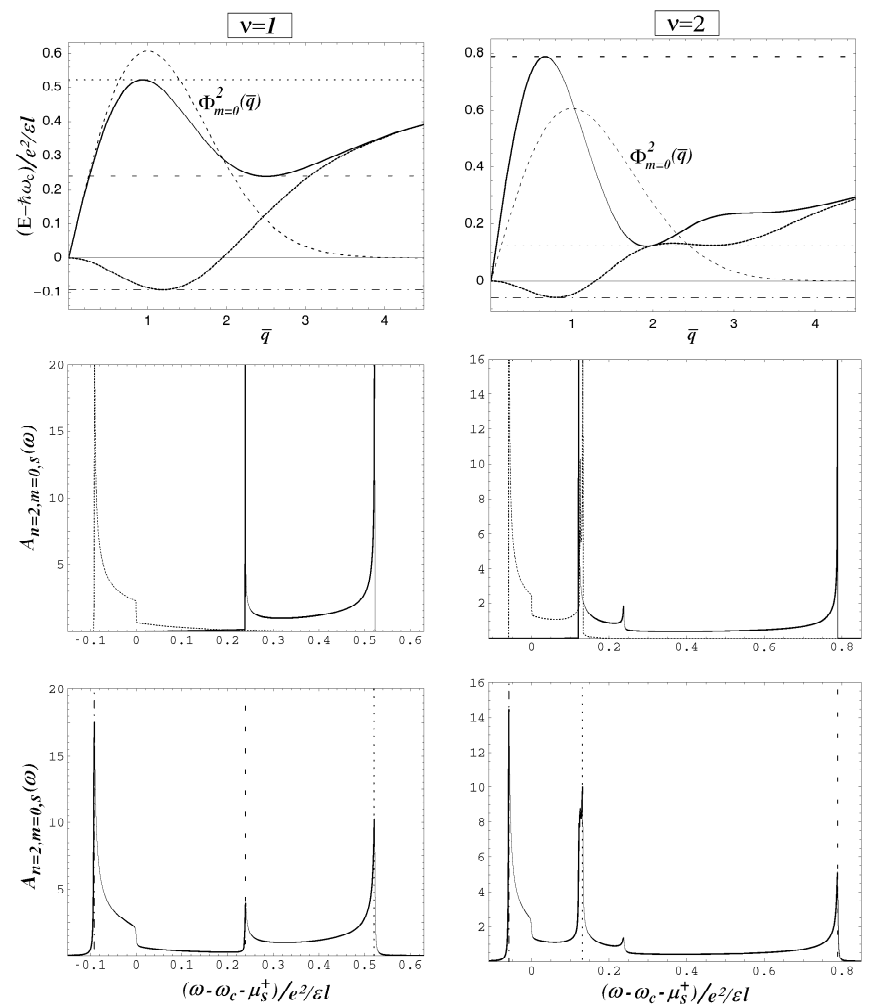

Figure 6: Spectral functions $A_{n=2, m=0, s}(\omega)$ for different filling factors $v$. In the second line the $\delta$-functions in $(7.23$ where substituted by Lorentzians of width $10^{-6} \omega_{c}$, stressing the integrable divergence at the local extremes of the dispersions $E_{n=1}^{(i)}(\bar{q})$. The charge (continuous) and spin (dotted) contributions are represented separately. The third line represents the addition of the two previous contributions, but with Lorentzians of width $10^{-3} \omega_{c}$, such that we can see the relative weight of the different peaks. Horizontal and vertical lines with the same dotted pattern correspond to the same energy $(\omega=E$ with $\hbar=1)$.

systems $^{25,38}$, for each non-interacting eigenstate, $|n, m, s\rangle$, we would obtain a single solution of the equation

$$
\omega-(n-1) \omega_{c}-\mu_{s}^{+}-\operatorname{Re} \Sigma_{r e t}\left(|n, m, s\rangle, \omega-\mu_{s}^{+}\right)=0,
$$

and this would be the energy of the quasi-particle in the interacting system. Here $\Sigma_{r e t}$ is the retarded self-energy ${ }^{25,38}$. However, in our case the above equation presents many solutions, one for each divergent peak in $A_{n, m, s}(\omega)$. So, in general, we see that we have a non-normal system. However, in our treatment, there are two exceptions. The first is the case associated with the inclusion of a particle in the lower allowed energy, when $A_{n=1, m, s}(\omega)$ is given by a simple $\delta$-function. The second is the case $A_{n, m \rightarrow N_{\Phi} \gg 1, s}(\omega)$, that we will see in the next section.

\section{F. Influence of the Guiding Center Position}

So far, in analyzing the spectral functions we have concentrated on the influence of the varying densities of states. How- ever, we have also mentioned the influence of the guiding center position, defined by $m$. The function $\Phi_{m}^{2}(\bar{q})$ weights the importance of the density of states at each point. The spectral functions presented in Figs.(5) and (6) were calculated with $m=0$. In this case $\Phi_{m=0}^{2}(\bar{q})$ does not present oscillations and limits the influence of the densities of states to the range of medium (not too large) $\bar{q}^{\prime} s$. However, once we increase the value of $m$, the function $\Phi_{m}^{2}(\bar{q})$ spreads and starts to present $m+1$ oscillations, with the last maximum being the largest one, see Fig.(7). Once the dispersion relations became softer at large $\bar{q}$ 's this region starts to concentrate most part of the spectral weight. Consequently, as shown in Fig.(7), the spectral function $A_{n=2, m, s}(\omega)$ starts to present weight at larger frequencies, with a peak much more pronounced that the one in $\Phi_{m}^{2}(\bar{q})$. In the limit $m \rightarrow N_{\Phi} \gg 1$ the peak will be concentrated at the asymptotic value $\omega_{c}+\mu_{s}^{+}+E_{S e l f}^{n=2}$, with a characteristic modulation. It is clear that, in this limit, more general spectral functions, $A_{n, m, s}(\omega)$, would also present themselves concentrated at well defined energies (shifted from the non-interacting values) and we will not see any effect of the decomposition into charge and spin fluctuations, once both present the same asymptotic limit. Systems presenting higher filling factors, as in Fig. (8), present a larger number of peaks, because of the $v$ oscillations in $E_{n}^{(\rho)}(\bar{q})$ and $E_{n}^{(\sigma)}(\bar{q})$, but their dispersions relations still converge to their asymptotic values in the limit $m \rightarrow N_{\Phi} \gg 1$.

We believe that this result can be understood as follows. A particle in a state $|n, m\rangle$ presents a probability density of width $\sqrt{2 n+1} l$, spread over a circle of radius $\sqrt{2 m+1} l$. Therefore, for not too large values of $n$, creating a particle in a state written in terms of $m \sim N_{\Phi}$ means to create a particle in a large radius, close to the edge of the system. Most of the electronhole pairs excited by the introduction of this particle should also spread over large radia, where their net wave function overlap is small and they do not fell much the presence of each other. Therefore, the energies excited in the system will be associated with electrons and holes far apart, $E_{\text {Self. }}^{n}$. However, the particles created close to the system's origin, with states written in terms of small $m$ 's, would present a much more complex structure in their spectral functions, because the electron-hole pairs excited will present a much larger correlation. Below, we will explicitly calculate the spectral function of a particle added at the system's origin.

The inclusion of the spin in $G^{\text {Ret }}(\mathbf{r}, t)$ is made by substituting $G_{n, m}^{R e t}(t)$ by $G_{n, m, s}^{R e t}(t)$ in (7.14):

$$
G^{R e t}(\mathbf{r}, t, s)=-i \sum_{n, m}|\langle\mathbf{r} \mid n, m\rangle|^{2} G_{n-(v-1), m, s}^{R e t}(t) .
$$

The same must be done for the calculation of $A(\mathbf{r}, s, \omega)$. For a particle added at the system's origin we have the expression equivalent to (7.21):

$$
A(\mathbf{r}=\mathbf{0}, s, \omega)=\frac{1}{(2 \pi l)^{2}} \sum_{n=0} A_{n-(v-1), n, s}(\omega) .
$$

Therefore, $A(\mathbf{r}=\mathbf{0}, s, \omega)$ will present many peaks. This is similar to the sum of the different peaks presented in Fig.(5), but with each peak being modulated by the corresponding 

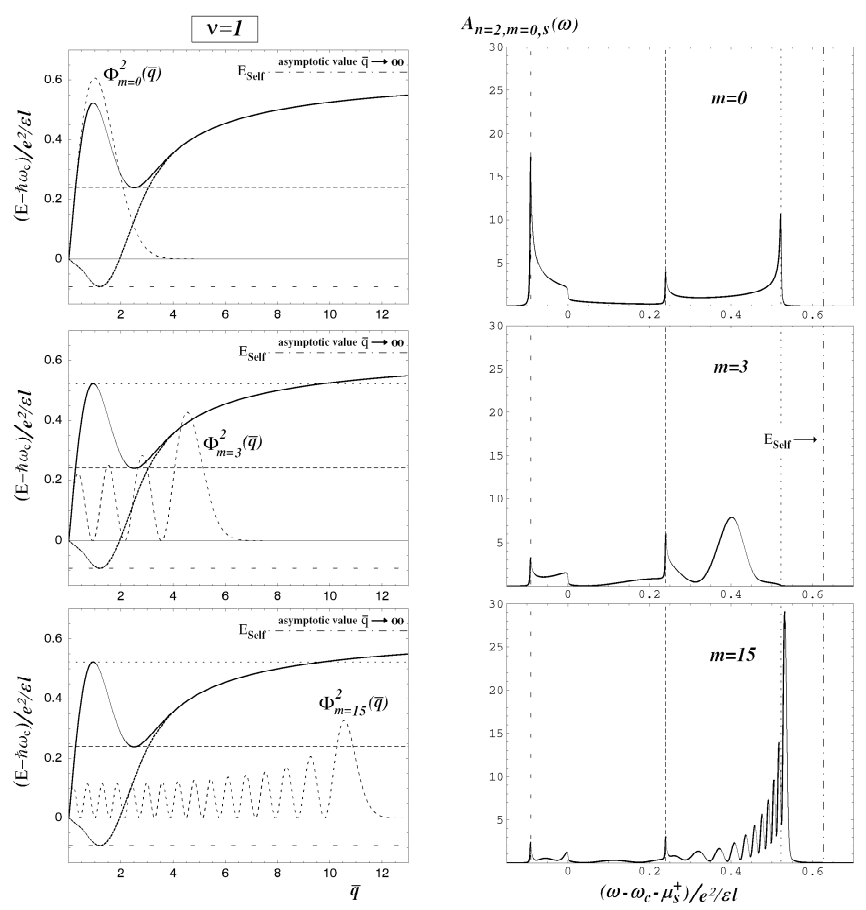

Figure 7: Spectral function $A_{n=2, m}(\omega)$ for $m=0,3$ and 15 , in a system presenting a filling factor $v=1$. Horizontal and vertical lines with the same dotted pattern correspond to the same energy. The vertical line indicated as $E_{S e l f}$ would be the position of the peak in the limit $m \rightarrow N_{\Phi} \gg 1$.

$\Phi_{m=n}^{2}(\bar{q})$ instead of the same $\Phi_{m=0}^{2}(\bar{q})$. The terms presenting $n \leq v-1$ will generate contributions to frequencies $\omega<0$, describing the effect of creating a hole in the system. They are similar to the ones in the region $\omega>0$. Fig.(9) presents the first two peaks of $A(\mathbf{r}=\mathbf{0}, s, \omega)$ in the region $\omega>0$ as an example.

Expression (7.26) also indicates that as the filling factor increases the spectral function develops better defined peaks in the $\omega>0$ region. In a system with $v \gg 1$ the spectral function $A(\mathbf{r}=\mathbf{0}, s, \omega)$ at frequencies $\omega \gtrsim \omega_{c}$ (associated with $\left.|n| \gtrsim v\right)$ will be governed by the functions $\Phi_{m=n \gtrsim v \gg 1}^{2}(\bar{q})$. So the system will tend to present a set of well defined peaks, shifted from the non-interacting values, as in a normal system.

\section{CONCLUSIONS}

In this work we presented a model and a method for the study of the integer quantum Hall systems presenting both spin sublevels filled $(\mathbf{S}=\mathbf{0})$. We showed that, to first order in $\gamma=\left(e^{2} / \varepsilon l\right) / \hbar \omega_{c}$, our model of interacting one-dimensional channels within the forward scattering approximation, which keeps constant the net number of particles in each channel, is equivalent to the strong field approximation. We saw how this model can be solved, with some well controlled approximations, through an appropriate variation of the Landau level bosonization method. Using this set of model and method we determined the collective excitations of the system: spin and
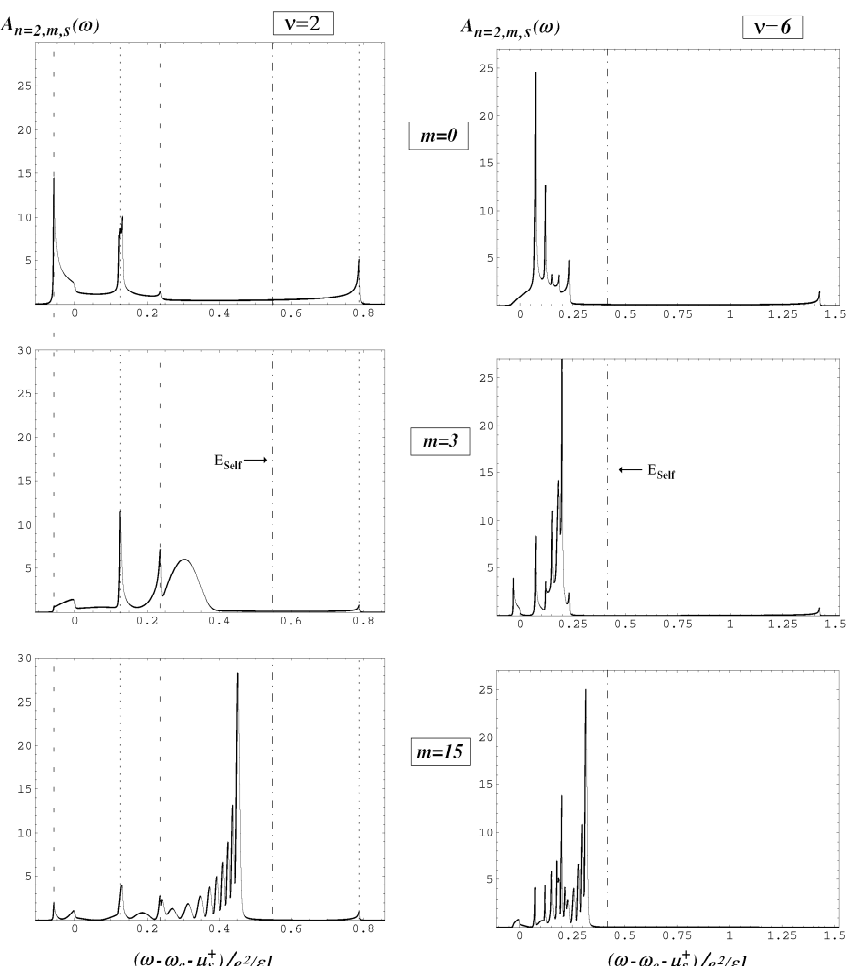

$\left(\omega-\omega_{c}-\mu_{s}^{+}\right) / e^{2 / \varepsilon l}$

$\left(\omega-\omega_{c}-\mu_{s}^{+}\right) / e^{2 / \varepsilon l}$

Figure 8: Spectral functions $A_{n=2, m, s}(\omega)$ for different $m$ values in a system presenting filling factors $v=2$ and $v=6$. The vertical dotted lines correspond to local extremes of the normal modes, as in the figure before, except for the vertical line indicated as $E_{S e l f}$, corresponding to the asymptotic value.

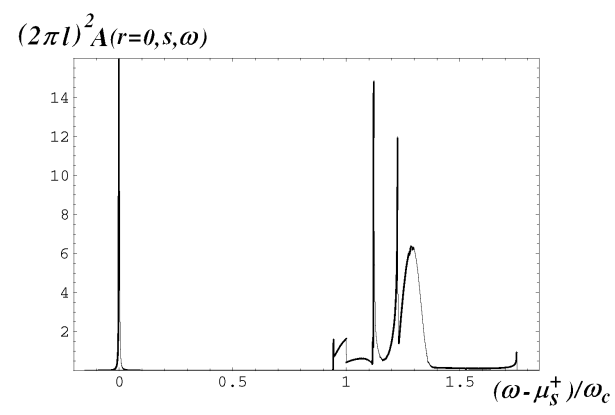

Figure 9: Spectral function for a particle created at the center of a GaAs heterojunction presenting filling factor $v=2$ under a transverse magnetic field $B=7 T$.

charge fluctuations, dependent on the direct and exchange interactions. We obtained the same dispersion relations as those presented by Kallin and Halperin $\frac{4}{*}$ and MacDonald $\underline{5}$ for the excitations close to the cyclotron frequency $\omega_{c}$.

From these non-monotonic dispersion relations and variable density of states, we determine the single particle spectral functions through the bosonic Mattis-Mandelstam representation of the fermionic operator. For states created close to the central region of the system, these functions presented the characteristic behavior of a strongly correlated non-normal system, including a spin and charge spectral decomposition 
and absence of quasi-particle excitations that keep similarities with the ones of a non-interacting system. However, for states close to the edge of the system these functions tend to present single peaks, shifted from the non-interacting values and with a characteristic envelope, like in a normal system. It is clear that, in our treatment, we do not consider the effects of the confining potential in the Hall system, which generate gapless edge excitations ${ }^{40}$ and are fundamental to a complete description of the edge states. However, independently of the additional effects of the confining potential, what we see is that the bulk quasi-particle states seem to be much more strongly coupled to the collective excitations in the system than the edge ones, which seems to behave like normal Fermi liquid particles, even in higher energies.

The experimental verification of the results we predicted seems to require a control over the state of the created particles higher than the one available in current experiments. However, if as we predicted here, the spectral functions for states created at the central region and those close to edge of the system have such a distinct behavior, this should not be too difficult to observe.

As we mentioned in the introduction and in Sec.(III.A), one of the advantages of the present model for the quantum Hall systems is that it presents a prescription for its improvement, once there are methods developed to treat the net particle transfer between neighbor channels in a bosonic description ${ }^{41}$. We saw that the previous perturbative methods for IQH systems ${ }^{4.5}$ used $\gamma$ as the expansion parameter. However, they do not give a systematic description, order by order, in this parameter. Indeed, not even the first order in $\gamma$ is fully described, indicating that this may not be the better expansion parameter. So, we propose, start from the forward scattering approximation, which is already a good initial description as we saw, and try to get higher order corrections taking the net particle transfer between " $m$ " neighbor channels as the expansion parameter. Once this transfer would be between channels with non-zero relative angular momentum, we suspect that the effective transfer potential can be related with some kind of Haldane pseudo-potential ${ }^{42}$, which, at least in the fractional quantum Hall systems, are known to be the really effective expansion parameters. Certainly, with the inclusion of the transfer between neighbor channels, we will have a Hamiltonian whose ground state will present particle-holes pairs, taking into account the Landau level mixing, which would be very convenient in many applications. In this case, the spectral functions $A_{n=1}(\omega)$ should already present a complex structure, in contrast to the $\delta$-function that we have got.

We also mentioned the fundamental importance of the collective modes in the FQHE, evidenced by the Hamiltonian theory of Shankar and Murthy ${ }^{11}$, which have many similarities with a bosonization method. Their fermionic field associated with the composite particle, $\psi_{C P}$, is very similar to a Mattis-Mandelstam representation associated with the magnetoplasmon excitation. This seems to us another evidence that systematic bosonization methods created for quantum Hall system should be explored.

Finally, we note that the one dimensional characteristics that seem to be retained in the 2 DEG under a strong perpen- dicular magnetic field, the influence of the collective modes in the quantum Hall quasi-particle properties and the possibility of using the relative angular momentum between particles as an expansion parameter in a field theory, probably can still be further explored in quantum Hall systems. Here we presented a method that partially explores the first two characteristics, indicates a way that can be followed in the investigation of the third one and can be improved for further applications.

\section{Acknowledgments}

MRC and AOC acknowledge Fundação de Amparo à Pesquisa do Estado de São Paulo (FAPESP) for the financial support. MRC also acknowledge Financiadora de Estudos e Projetos (FINEP) and Ministério da Ciência e Tecnologia (MCT). HW and AOC also acknowledge Conselho Nacional de Desenvolvimento Científico e Tecnológico $(\mathrm{CNPq})$ for partial support.

\section{Appendix A: SOME PROPERTIES OF THE FUNCTIONS $G_{n, m^{\prime}}(\mathbf{q})$}

In this Appendix we just list some relations among the $G_{n, m}(\mathbf{q})$ functions $^{21}$, with $\mathbf{q}=q_{x}+i q_{y}$, which are useful for the matrix element manipulation.

Hermitian Conjugate:

$$
G_{m^{\prime}, m}(\mathbf{q})=G_{m, m^{\prime}}^{*}(-\mathbf{q})=G_{m, m^{\prime}}\left(\mathbf{q}^{*}\right) .
$$

Matrix Product:

$$
\sum_{l} G_{m^{\prime}, l}(\mathbf{q}) G_{l, m}\left(\mathbf{q}^{\prime}\right)=e^{-\frac{\mathbf{q}^{*} \mathbf{q}^{\prime}}{2}} G_{m^{\prime}, m}\left(\mathbf{q}+\mathbf{q}^{\prime}\right) .
$$

Full Landau Level Rule:

$$
\sum_{m=0}^{\infty} G_{m \cdot m}(\mathbf{q})=N_{\Phi} \delta_{\mathbf{q}, \mathbf{0}}=\frac{(2 \pi)^{2}}{S} N_{\Phi} \delta(\mathbf{q})
$$

Orthogonality:

$$
\int d^{2} \mathbf{q} e^{-q^{2} / 2} G_{m \cdot m^{\prime}}(\mathbf{q}) G_{n, n^{\prime}}\left(\mathbf{q}^{*}\right)=\frac{1}{2 \pi} \delta_{m, n} \delta_{m^{\prime}, n^{\prime}} .
$$

Matrix Elements:

$$
\left\langle n, m\left|e^{-i \mathbf{q} \cdot \mathbf{r}}\right| n^{\prime}, m^{\prime}\right\rangle=e^{-\frac{\mid l \mathbf{q}^{2}}{2}} G_{n, n^{\prime}}\left(l \mathbf{q}^{*}\right) G_{m, m^{\prime}}(l \mathbf{q}) .
$$

\section{Appendix B: THE INTERACTION MATRIX ELEMENTS}

To obtain the matrix elements of the interaction Hamiltonian in the forward scattering approximation, eq.(3.4), one has to pair the creation and annihilation fermionic operators of (2.6) in all possible ways and set the same guiding center 
$m$ for both. This is easily accomplished by defining a generalized density operator

$$
\rho(\mathbf{q}, \mathbf{x})=e^{-\frac{\bar{q}^{2}}{2}} \sum_{\{n, m\}} G_{n, n^{\prime}}\left(\overline{\mathbf{Q}}^{*}\right) G_{m, m^{\prime}}(\overline{\mathbf{q}}) c_{n, m}^{\dagger} c_{n^{\prime}, m^{\prime}},
$$

with $\overline{\mathbf{q}}=\mathbf{q} l, \overline{\mathbf{Q}}=\overline{\mathbf{q}}+i \overline{\mathbf{x}}$ and $\overline{\mathbf{x}}=\mathbf{x} / l$, and finding its bosonic representation in terms of the operators $b_{n, m}$ or even directly in terms of $b_{n}(q)$, as we will do here. It is straightforward, though a little cumbersome, to show that the two ways of pairing the creation and annihilation operators of the Hamiltonian that yield the direct and exchange terms as follows

$$
\begin{aligned}
& H_{I}^{D i r}=\frac{1}{2} \frac{1}{(2 \pi)^{2}} \int d^{2} q \tilde{V}(\mathbf{q}) \rho^{\dagger}(\mathbf{q}) \rho(\mathbf{q})-\frac{1}{2} V(\mathbf{0}) \hat{N}_{T}, \\
& H_{I}^{E x}=-\frac{1}{2} \frac{1}{(2 \pi)^{2}} \int d^{2} q \int d^{2} x V(x) e^{-\frac{\bar{x}^{2}}{2}} e^{i\left(\overline{\mathbf{q}} \cdot \overline{\mathbf{x}}^{*}-\overline{\mathbf{q}}^{*} \cdot \overline{\mathbf{x}}\right) / 2} \\
& \times \rho(\mathbf{q}, \mathbf{x}) \rho^{\dagger}(\mathbf{q}, \mathbf{x})+\frac{1}{2 S} \tilde{V}(0) \hat{N}_{T},
\end{aligned}
$$

where $\rho(\mathbf{q})=\rho(\mathbf{q}, \mathbf{x}=\mathbf{0})$ is the electron density operator and we introduced the total particle number operator $\hat{N}_{T}=$ $\sum_{n, m} c_{n, m}^{\dagger} c_{n, m}$. To obtain the exchange term ${ }^{26}$, the matrix element in 2.6 must be expressed in the position basis, $\left|\mathbf{r}, \mathbf{r}^{\prime}\right\rangle$, using (2.3). Then, in the resulting expression, we replace the integration variables, $\mathbf{r}$ and $\mathbf{r}^{\prime}$, by the relative, $\mathbf{x}=\mathbf{r}-\mathbf{r}^{\prime}$, and center of mass, $\mathbf{X}=\left(\mathbf{r}+\mathbf{r}^{\prime}\right) / 2$, coordinates evaluate the integral with respect to the latter and use the relation (A2) from Appendix A.

To obtain the bosonic representation of $\rho(\mathbf{q}, \mathbf{x})$ we first rewrite the sum over $\left\{n, n^{\prime}\right\}$ as $\sum_{n, n^{\prime}}=\sum_{n>n^{\prime}}+\sum_{n<n^{\prime}}+(n=$ $\left.n^{\prime}\right)$, doing $n \equiv p+n$ and $n^{\prime} \equiv n$ in the terms where $n>n^{\prime}$ and $n \rightleftarrows n^{\prime}$ when $n<n^{\prime}$. At the same time we can make the forward scattering approximation, $m=m^{\prime}$, to get

$$
\begin{aligned}
\rho(\mathbf{q}, \mathbf{x})= & e^{-\frac{\bar{q}^{2}}{2}} \sum_{n>0, p}\left[G_{p, p+n}\left(\overline{\mathbf{Q}}^{*}\right) \sum_{m} G_{m, m}(\overline{\mathbf{q}}) c_{p, m}^{\dagger} c_{p+n, m}\right. \\
& \left.+G_{p+n, p}\left(\overline{\mathbf{Q}}^{*}\right) \sum_{m} G_{m, m}(\overline{\mathbf{q}}) c_{p+n, m}^{\dagger} c_{p, m}\right] \quad(\mathrm{B} 4) \\
& +\hat{\rho}_{N}(\mathbf{q}, \mathbf{x}),
\end{aligned}
$$

where

$$
\hat{\rho}_{N}(\mathbf{q}, \mathbf{x})=e^{-\frac{\bar{q}^{2}}{2}} \sum_{n, m} G_{n, n}\left(\overline{\mathbf{Q}}^{*}\right) G_{m, m}(\overline{\mathbf{q}}) c_{n, m}^{\dagger} c_{n, m}
$$

Now we approximate $\left.G_{p, p+n}\left(\overline{\mathbf{Q}}^{*}\right) \simeq G_{p, p+n}\left(\overline{\mathbf{Q}}^{*}\right)\right|_{p=v-1}$ since we are restricted to the low energy excitations close to the higher filled Landau level, $v-1$. Using simultaneously (3.1) and (3.11), we get:

$$
\begin{aligned}
\rho(\mathbf{q}, \mathbf{x})= & \frac{e^{-\frac{\bar{q}^{2}}{4}}}{\sqrt{\bar{q}}} \sum_{n} \sqrt{n}\left[G_{\boldsymbol{v}-1, v-1+n}\left(\overline{\mathbf{Q}}^{*}\right) b_{n}(\bar{q})\right. \\
& \left.+G_{v-1+n, v-1}\left(\overline{\mathbf{Q}}^{*}\right) b_{n}^{\dagger}(\bar{q})\right]+\hat{\rho}_{N}(\mathbf{q}, \mathbf{x}) .
\end{aligned}
$$

The bosonization of $\hat{\rho}_{N}(\mathbf{q}, \mathbf{x})$ is done in the same way as the bosonization of $H_{0}{ }^{18}$, eq. (3.3), because both have the same form except for the expression $G_{n, n}(\overline{\mathbf{Q}}) G_{m, m}(\overline{\mathbf{q}})$ which replaces the simpler function $n$. Calculating the commutator

$$
\begin{aligned}
{\left[\hat{\boldsymbol{\rho}}_{N}(\mathbf{q}, \mathbf{x}), b_{N, M}\right] } & =G_{M, M}(\overline{\mathbf{q}}) \frac{e^{-\frac{\bar{q}^{2}}{2}}}{\sqrt{N}} \sum_{p}\left[G_{p, p}\left(\overline{\mathbf{Q}}^{*}\right)-G_{p+N, p+N}\left(\overline{\mathbf{Q}}^{*}\right)\right] c_{p, M}^{\dagger} c_{p+N, M} \\
& \simeq e^{-\frac{\bar{q}^{2}}{2}} L_{M}\left(\frac{\bar{q}^{2}}{2}\right)\left[L_{v-1}\left(\frac{\bar{Q}^{2}}{2}\right)-L_{v-1+N}\left(\frac{\bar{Q}^{2}}{2}\right)\right] b_{N, M}
\end{aligned}
$$

we finally conclude that $\hat{\rho}_{N}(\mathbf{q}, \mathbf{x})$ must have the following bosonic representation:

$$
\hat{\rho}_{N}(\mathbf{q}, \mathbf{x})=\left\langle G_{\mathbf{0}}\left|\hat{\rho}_{N}(\mathbf{q}, \mathbf{x})\right| G_{\mathbf{0}}\right\rangle+e^{-\frac{\bar{q}^{2}}{2}} \sum_{n, m} L_{m}\left(\frac{\bar{q}^{2}}{2}\right)\left[L_{v-1}\left(\frac{\bar{Q}^{2}}{2}\right)-L_{v-1+n}\left(\frac{\bar{Q}^{2}}{2}\right)\right] b_{n, m}^{\dagger} b_{n, m},
$$

where

$$
\left\langle G_{\mathbf{0}}\left|\hat{\rho}_{N}(\mathbf{q}, \mathbf{x})\right| G_{\mathbf{0}}\right\rangle=\sum_{n=0}^{v-1} G_{n, n}\left(l \mathbf{q}^{*}-i \mathbf{x}^{*} / l\right) \sum_{m=0}^{N_{\Phi}} G_{m, m}(l \mathbf{q})=\frac{2 \pi}{l^{2}} L_{v-1}^{1}\left(\frac{x^{2}}{2 l^{2}}\right) \delta(\mathbf{q})
$$

In the last step we have used the relations $\mathrm{A3}$ and $\sum_{m=0}^{n} L_{m}^{\alpha}(x)=L_{n}^{\alpha+1}(x)$.

The bosonic representation of $\rho(\mathbf{q})$ is a particular case of relation (B1) and is given by

$$
\rho(\mathbf{q})=\frac{e^{-\frac{\bar{q}^{2}}{4}}}{\sqrt{\bar{q}}} \sum_{n>0} \sqrt{n}\left[G_{v-1, v-1+n}\left(\overline{\mathbf{q}}^{*}\right) b_{n}(\bar{q})+G_{v-1+n, v-1}\left(\overline{\mathbf{q}}^{*}\right) b_{n}^{\dagger}(\bar{q})\right]+\hat{\rho}_{N}(\mathbf{q}) .
$$


The last term, $\hat{\rho}_{N}(\mathbf{q})=\hat{\rho}_{N}(\mathbf{q}, \mathbf{0})$, will give a null contribution after its substitution in (B2) if we neglect cubic terms in the boson operators. These would describe the dissociation of an electron-hole pair 19 .

Substituting (B8) in (B) electron density $\left\langle G_{\mathbf{0}}\left|\hat{\rho}_{N}(\mathbf{q})\right| G_{\mathbf{0}}\right\rangle=\frac{2 \pi v}{l^{2}} \delta(\mathbf{q})$ present in $\hat{\rho}_{N}(\mathbf{q})=\hat{\rho}_{N}(\mathbf{q}, \mathbf{0})$, eq.(B6) $)$ and performing the angular integration over $\arg (\mathbf{q})$ (that eliminates the non-diagonal quadratic terms, see section $\amalg$ IIC), we finally obtain the direct term of (3.4).

Now, following the same procedure and substituting the bosonic representation, (B5) and (B6), of $\rho(\mathbf{q}, \mathbf{x})$ in (B3) we verify that, after the integration over $\arg (\mathbf{x})$, the linear terms in the boson operators present null coefficients. If we neglect cubic terms in the boson operators, we get $H_{I}^{E x}$ written as:

$$
\begin{gathered}
H_{I}^{E x}=H_{\text {Col }}^{E x}+H_{\text {Self }}^{E x}, \\
H_{C o l}^{E x}=-\frac{1}{2(2 \pi)^{2}} \int d^{2} q \frac{e^{-\frac{\bar{q}^{2}}{2}}}{\bar{q}} \int d^{2} x V(x) e^{-\frac{\bar{x}^{2}}{2}} e^{i\left(\overline{\mathbf{q}} \cdot \overline{\mathbf{x}}^{*}-\overline{\mathbf{q}}^{*} \cdot \overline{\mathbf{x}}\right) / 2} \\
\times\left\{\sum_{n>0} \sqrt{n}\left[G_{v-1, v-1+n}\left(\overline{\mathbf{Q}}^{*}\right) b_{n}(\bar{q})+G_{v-1+n, v-1}\left(\overline{\mathbf{Q}}^{*}\right) b_{n}^{\dagger}(\bar{q})\right]\right\} \times\{h . c .\}, \\
H_{\text {Self }}^{E x}=\frac{1}{2 \pi l^{2}} \int d^{2} x V(x) e^{-\frac{\bar{x}^{2}}{2}} L_{v-1}^{1}\left(\frac{\bar{x}^{2}}{2}\right) \sum_{n>0}\left[L_{v-1}\left(\frac{\bar{x}^{2}}{2}\right)-L_{v-1+n}\left(\frac{\bar{x}^{2}}{2}\right)\right] \int_{0}^{\infty} d \bar{q} b_{n}^{\dagger}(\bar{q}) b_{n}(\bar{q}) .
\end{gathered}
$$

Expanding eq. (B9) we will have different exchange effective potentials, $v_{n n^{\prime}}^{E x}(\mathbf{q})$, associated with the different combinations of operators $b_{n}$ and $b_{n^{\prime}}^{\dagger}$ obtained. For example, the one associated with the combination $b_{n}(\bar{q}) b_{n^{\prime}}^{\dagger}(\bar{q})$ is given by:

$$
v_{n n^{\prime}}^{E x}(\mathbf{q})=-\frac{\sqrt{n n^{\prime}}}{2(2 \pi)^{2}} \frac{e^{-\bar{q} / 2}}{\bar{q}} \int d^{2} x V(x) e^{-\frac{\bar{z}^{2}}{2}} e^{i\left(\overline{\mathbf{q}} \cdot \overline{\mathbf{x}}^{*}-\overline{\mathbf{q}} \cdot \overline{\mathbf{x}}\right) / 2} G_{v-1, v-1+n}\left(\overline{\mathbf{Q}}^{*}\right) G_{v-1, v-1+n^{\prime}}^{*}\left(\overline{\mathbf{Q}}^{*}\right) .
$$

The integration variable, $\mathbf{x}$, is different from the variable of the integrand, $\overline{\mathbf{Q}}^{*}=\overline{\mathbf{q}}^{*}-i \overline{\mathbf{x}}^{*}$. However, if we decompose the function $G(\overline{\mathbf{Q}})$ using the matrix product $\underline{\mathrm{A} 2}$, apply the Fourier transform,

$$
e^{-\bar{x}^{2} / 2} G_{m, n}(-i \overline{\mathbf{x}}) G_{m^{\prime}, n^{\prime}}^{*}\left(-i \overline{\mathbf{x}}^{*}\right)=\frac{1}{2 \pi} \int d^{2} \bar{k} e^{-i \overline{\mathbf{k}} \cdot \overline{\mathbf{x}}} G_{n^{\prime}, n}\left(\overline{\mathbf{k}}^{*}\right) G_{m^{\prime}, m}(\overline{\mathbf{k}}) e^{-\bar{k}^{2} / 2}
$$

realize the integration over $\overline{\mathbf{x}}$ and, finally, contract the $G$ function using (A2) again, we get:

$$
v_{n n^{\prime}}^{E x}(\mathbf{q})=-\frac{\sqrt{n n^{\prime}}}{2(2 \pi)^{3}} \frac{1}{\bar{q}} \int d^{2} \bar{k} \tilde{V}(k) e^{-\bar{k}^{2} / 2} e^{\left(\overline{\mathbf{q}} \cdot \overline{\mathbf{k}}^{*}-\overline{\mathbf{k}} \cdot \overline{\mathbf{q}}^{*}\right) / 2} G_{v-1+n^{\prime}, v-1+n}\left(\overline{\mathbf{k}}^{*}\right) G_{v-1, v-1}\left(\overline{\mathbf{k}}^{*}\right) .
$$

The angular portion of this integral, $\overline{\mathbf{k}}=\bar{k} e^{i \theta_{\mathbf{k}}}$, is given by:

$$
\int d \theta_{\mathbf{k}} e^{i \bar{k}\left(\cos \theta_{k} \bar{q}_{y}-\sin \theta_{k} \bar{q}_{x}\right)} e^{i \theta_{\mathbf{k}}\left(n-n^{\prime}\right)}=2 \pi g_{n^{\prime}-n}(\bar{k} \bar{q}) e^{i \theta_{\mathbf{q}}\left(n^{\prime}-n\right)},
$$

where we use eq.(3.937) from Ref.[39] and $I_{n}$ is the Bessel function of order $n$. Now, due the presence of the coefficient $e^{i \theta_{\mathbf{q}}\left(n^{\prime}-n\right)}$ in the expression above, only the terms $n=n^{\prime}$ will survive after the angular integration over $\arg (\mathbf{q})=\theta_{\mathbf{q}}$ in (B9). Therefore, we will need only

$$
\begin{aligned}
v_{n=n^{\prime}}^{E x}(\mathbf{q})= & -\frac{n}{2(2 \pi)^{2}} \frac{1}{\bar{q}} \int d \bar{k} \bar{k} \tilde{V}(k) e^{-\bar{k}^{2} / 2} \\
& \times L_{v-1+n}\left(\frac{\bar{k}^{2}}{2}\right) L_{v-1}\left(\frac{\bar{k}^{2}}{2}\right) g_{0}(\bar{k} \bar{q}) .
\end{aligned}
$$

Repeating the same procedure as above to the other coefficients, we verify that the ones associated with the terms $b_{n}(q) b_{n^{\prime}}(q)$ and $b_{n}^{\dagger}(q) b_{n^{\prime}}^{\dagger}(q)$ will be proportional to $e^{ \pm i \theta_{\mathbf{q}}\left(n^{\prime}+n\right)}$, instead of $e^{i \theta_{q}\left(n^{\prime}-n\right)}$ as in (B12). Therefore, once $n, n^{\prime}>0$, they will disappear after the angular integration over $\theta_{\mathbf{q}}$ in (B9). The coefficient associated with $b_{n}^{\dagger}(q) b_{n^{\prime}}(q)$ will be the same as the one in B13). In this way, remembering that $\tilde{V}(k)=2 \pi e^{2} / \varepsilon k$, we get the expressions (3.6) to (3.9) .

\section{Appendix C: CALCULATION OF THE FUNCTIONS $G_{m, \theta}^{\gtrless}(t)$}

In this Appendix we present the calculation of the Green's functions $G_{m, \theta}^{\gtrless}(t)$, defined in (7.10). The method used is the common one in bosonization procedures. We suggest Ref.[16] 
for a review. We have

$$
\begin{aligned}
i G_{m, \theta}^{>}(t) & =\left\langle\psi_{m}(\theta, t) \psi_{m}^{\dagger}(0,0)\right\rangle_{0} \\
& =\lim _{\varepsilon \rightarrow 0} \frac{1}{2 \pi \varepsilon}\left\langle U_{m}(t) e^{-i \hat{N}_{m} \theta} e^{-i \Theta_{m}(\theta, t)} e^{i \Theta_{m}(0,0)} U_{m}^{\dagger}\right\rangle_{0} \\
& =\lim _{\varepsilon \rightarrow 0} \frac{1}{2 \pi \varepsilon} e^{-i\left(\mu_{m}^{+} t+\theta\right)}\left\langle e^{-i \Theta_{m}(\theta, t)} e^{i \Theta_{m}(0,0)}\right\rangle_{0},
\end{aligned}
$$

where we use (7.8). So, we just have to calculate the propagator

$$
K_{m, \theta}(t)=\lim _{\varepsilon \rightarrow 0} \frac{1}{2 \pi \varepsilon}\left\langle e^{-i \Theta_{m}(\theta, t)} e^{i \Theta_{m}(0,0)}\right\rangle_{0} .
$$

Using the identity $e^{A} e^{B}=e^{B} e^{A} e^{C}$, where $C=[A, B] / 2$ and $[C, A]=[C, B]=0$, we can write the above expression in a normal ordered form, or use directly the relation

$$
\left\langle e^{\lambda_{1} \beta_{1}} e^{\lambda_{2} \beta_{2}}\right\rangle_{0}=e^{\left\langle\lambda_{1} \beta_{1} \lambda_{2} \beta_{2}+\frac{1}{2}\left(\lambda_{1}^{2} \beta_{1}^{2}+\lambda_{2}^{2} \beta_{2}^{2}\right)\right\rangle_{0}}
$$

valid for bosonic operators $\beta$ acting on eigenstates of a free boson Hamiltonian ${ }^{18}$, and we get:

$$
K_{m}(\theta, t)=\lim _{\varepsilon \rightarrow 0} \frac{1}{2 \pi \varepsilon} e^{J_{m}(\theta, t)},
$$

where

$$
J_{m}(\theta, t)=D_{m}(\theta, t)-D_{m}(0,0)
$$

and

$$
\begin{aligned}
D_{m}(\theta, t) & =\left\langle\Theta_{m}(\theta, t) \Theta_{m}(0,0)\right\rangle \\
& =\int_{0}^{\infty} d \bar{q} \Phi_{m}^{2}(\bar{q}) \sum_{n=1}^{\infty} \frac{e^{-n \varepsilon}}{n} e^{-i n \theta} e^{-i E_{n}(\bar{q}) t} .
\end{aligned}
$$

Here we have used the expression (7.7) of $\Theta_{m}(\theta, t)$. Therefore,

$$
\begin{aligned}
i G_{m, \theta}^{>}(t)= & \lim _{\varepsilon \rightarrow 0} \frac{1}{2 \pi \varepsilon} e^{-i\left(\mu_{m}^{+} t+\theta\right)} \\
& \times e^{\int_{0}^{\infty} d \bar{q} \Phi_{m}^{2}(\bar{q}) \sum_{n=1}^{\infty} \frac{e^{-n \varepsilon}}{n}\left[e^{-i n \theta} e^{-i E_{n}(\bar{q}) t}-1\right]} \\
= & \frac{1}{2 \pi} e^{-i\left(\mu_{m}^{+} t+\theta\right)} e^{\int_{0}^{\infty} d \bar{q} \Phi_{m}^{2}(\bar{q}) \sum_{n=1}^{\infty} \frac{e^{-n \varepsilon}}{n} e^{-i n \theta} e^{-i E_{n}(\bar{q}) t}},
\end{aligned}
$$

where we use $\ln (1-x)=-\sum_{n=1}^{\infty} x^{n} / n$, with $x=e^{-\varepsilon}$, in the last equation. The calculation of $G_{m, \theta}^{<}(t)$ is analogous to the one presented above.

\section{Appendix D: CALCULATION OF THE FUNCTIONS $G_{m, \theta, s}^{\gtrless}(t)$}

Including the electron spin, the fermionic operator evolves in time as

$$
\begin{aligned}
\psi_{m, s}(\theta, t)= & \lim _{\varepsilon \rightarrow 0} \frac{1}{\sqrt{2 \pi \varepsilon}} U_{m, s}(t) e^{-i \hat{N}_{m, s} \theta} e^{-i \Theta_{m, s}(\theta, t)}, \\
\Theta_{m, s}(\theta, t)= & i \sum_{n=1}^{\infty} \frac{e^{-n \varepsilon / 2}}{\sqrt{n}} \int_{0}^{\infty} d \bar{q} \Phi_{m}(\bar{q}) \\
& \times\left[e^{-i n \theta} b_{n, s}(\bar{q}, t)-e^{i n \theta} b_{n, s}^{\dagger}(\bar{q}, t)\right]
\end{aligned}
$$

Using (5.4) and (5.5) we can express the operators $b_{n, s}(\bar{q})$ in terms of the charge and spin bosonic operators, $b_{n}(\bar{q})_{\rho}$ and $b_{n}(\bar{q})_{\sigma}$. Then we will have

$$
\begin{aligned}
\Theta_{m, s}(\theta, t)= & -\frac{i}{\sqrt{2}} \sum_{n=1}^{\infty} \frac{e^{-n \varepsilon / 2}}{\sqrt{n}} \int_{0}^{\infty} d \bar{q} \Phi_{m}(\bar{q}) \\
& \times\left\{e^{i n \theta} b_{n}^{\dagger}(\bar{q}, t)_{\rho}-e^{-i n \theta} b_{n}(\bar{q}, t)_{\rho}\right. \\
& \left.+s\left[e^{i n \theta} b_{n}^{\dagger}(\bar{q}, t)_{\sigma}-e^{-i n \theta} b_{n}(\bar{q}, t)_{\sigma}\right]\right\}
\end{aligned}
$$

Due the separation of the total Hamiltonian $H_{T}$ in the charge and spin modes (see (5.7) we will have the trivial evolution, $b_{n}(\bar{q}, t)_{i}=e^{-i E_{n}^{(i)}(\bar{q}) t} b_{n}(\bar{q})_{i}$, with $E_{n}^{(i)}(\bar{q})$ given by (5.8).

Now, the retarded Green's function associated with the inclusion of a particle in the quantum state $|n, m, s\rangle$ is given by:

$$
\begin{aligned}
i G_{n, m, s}^{R e t}(t)= & \theta(t)\left\langle c_{n, m, s}(t) c_{n, m, s}^{\dagger}(0)\right. \\
& \left.+c_{n, m, s}^{\dagger}(0) c_{n, m, s}(t)\right\rangle \\
= & i \theta(t) \int_{0}^{2 \pi} d \theta e^{i n \theta}\left[G_{m, s, \theta}^{>}(t)+G_{m, s, \theta}^{<}(t)\right],
\end{aligned}
$$

where

$$
\begin{aligned}
i G_{m, s, \theta}^{>}(t) & =\left\langle\psi_{m, s}(\theta, t) \psi_{m, s}^{\dagger}(0,0)\right\rangle, \\
i G_{m, s, \theta}^{<}(t) & =\left\langle\psi_{m, s}^{\dagger}(0,0) \psi_{m, s}(\theta, t)\right\rangle .
\end{aligned}
$$

Now, due the commutativity between the operators $b_{n}^{(\dagger)}(\bar{q}, t)_{\rho}$ and $b_{n}^{(\dagger)}(\bar{q}, t)_{\sigma}$ and the separation of the total Hamiltonian in the form (5.7), we have the characteristic factorization $^{27,28}$ of the operators $G_{m, s, \theta}^{\gtrless}(t)$ into charge and spin propagators. For example:

$$
\begin{aligned}
i G_{m, s, \theta}^{>}(t) & =e^{-i \mu_{s}^{+} t} e^{-i \theta} K_{m, \rho, \theta}(t) K_{m, \sigma, \theta}(t) \\
K_{m, i, \theta}(t) & =\frac{1}{\sqrt{2 \pi}} e^{\frac{1}{2} \sum_{n=1}^{\infty} \frac{e^{-n \varepsilon / 2}}{n} e^{-i n \theta} \int_{0}^{\infty} d \bar{q} \Phi_{m}^{2}(\bar{q}) e^{-i E_{n}^{(i)}(\bar{q}) t}}
\end{aligned}
$$

where the factor $1 / 2$ in the exponent comes from the factor $1 / \sqrt{2}$ in the definitions (5.4) and (5.5) of $b_{n}(\bar{q}, t)_{i}$. Calculating $i G_{m, \theta}^{<}(t)$ in a analogous way and substituting in (D1) we get 7.22 , with

$$
M_{m, \theta}(t)=K_{m, \rho, \theta}(t) K_{m, \sigma, \theta}(t) .
$$


1 For a review, see "The Quantum Hall Effect", edited by R. E. Prange and S. M. Girvin (Springer-Verlag, New York, 1987).

2 R. B. Laughlin, Rev. of Mod. Phys. 71, 863 (1999) and references therein.

${ }^{3}$ For a review, see "Perspectives in Quantum Hall Effects", edited by S. das Sarma and A. Pinczuk (John Wiley \& Sons, New York, 1997).

4 C. Kallin and B. I. Halperin, Phys. Rev. B, 30, 5655 (1984).

5 A. H. MacDonald, J. Phys. C, 18, 1003 (1985).

6 C. Kallin in "Interfaces, Quantum Wells and Superlatices", Ed. C. R. Leavens and R. Taylor, Plenum, New York (1988).

7 A. Pinkzuk et al., Phys. Rev. Lett. 61, 2701 (1988). A. Pinczuk in Ref.[3] and references therein.

8 U. Zeitler et al., Phys. Rev. Lett. 82, 5333 (1999).

9 V. M. Apalkov and M. E. Portnoi, Phys. Rev. B 66, 121303R (2002).

10 M. A. Eriksson et al., Phys. Rev. Lett. 82, 2163 (1999).

11 G. Murthy and R. Shankar, Rev. of Mod. Phys. 75, 1101 (2003).

12 J. K. Jain, Phys. Rev. Lett. 63, 199 (1989); Adv. Phys. 41, 105 (1992).

13 For a review, see "Composite Fermions", edited by O. Heinonen (World Scietific, Teaneck, 1998).

14 B. I. Halperin, P. A. Lee and N. Read, Phys. Rev. B 47, 7312 (1993).

15 M. Rosenau da Costa, H. Westfahl and A. O. Caldeira to be published.

16 H. Westfahl Jr., A. H. Castro Neto and A. O. Caldeira, Phys. Rev. B 55, R7347 (1997). H. Westfahl Jr., A. H. Castro Neto and A. O. Caldeira, Zeits. Für Physick B 103, 279 (1997).

17 S. Mandelstam , Phys. Rev. D 11,3026 (1975).

18 J. Van Delft and H. Schoeller, Annalen Phys.7, 225 (1998).

19 S. Cheng, Phys. Rev. B 49, 4703 (1994).

20 A. H. Castro Neto and E. Fradkin, Phys. Rev. Lett. 72, 1393 (1993); Phys. Rev. B, 51, 4084 (1995).

21 A. H. MacDonald, in Les Houches, Session LXI, "Physique Quantique Mesoscopique", edited by E. Akkermans, G. Montambeaux and J.L. Pichard (Elsevier, Amsterdam, 1995).
22 A. Houghton and B. Marston, Phys. Rev. B 48, 7790 (1993); H. J. Kwon, A. Houghton and B. Marston, Phys. Rev. B 52, 8002 (1995). A. Houghton, H. J. Kwon and J.B. Marston, Adv. in Phys., 49, 141 (2000).

23 A. Luther, Phys. Rev. B, 19, 320 (1979).

24 J. M. Luttinger, J. Math. Phys. 15, 609 (1963).

25 G. D. Mahan, "Many Particle Physics" (Plenum Press, 1981).

26 H. Backes, undergraduate thesis.

27 J. Voit, Rep. Prog. Phys. 58, 977 (1995) and references therein.

28 K. Schonhammer, Jour. of Phys. - Cond. Mat. 14, 12783 (2002).

29 A. Luther and V. J. Emery, Phys. Rev. Lett. 33, 589 (1974).

30 V. Meden and K. Shönhammer, Phys. Rev. B 46, 15753 (1992).

31 J. P. Longo and C. Kallin, Phys. Rev. B 47, 4429 (1993).

32 R. L. Doretto, A. O. Caldeira and S. M. Girvin, Phys. Rev. B 71, 045339 (2005).

33 When we say $v=1$ we means, in the Kallin and Halperin notation, $v_{\downarrow}=v_{\uparrow}=1$, once we are always considering the two spin states (Landau sublevels) initially equally occupied.

34 C. Kallin and B. I. Halperin, Phys. Rev. B, 31, 3635 (1985).

35 F. D. M. Haldane, J. Phys. C 14, 2585 (1981).

36 F. Anfuso, S. Eggert, Phys. Rev. B 68, 241301(R) (2003).

37 J. Voit, J. Phys. C 5, 8305 (1993); J. Voit, Phys. Rev. B 47, 6740 (1993).

38 P. Nozières, "Theory of Interacting Fermi Systens ", W. A. Benjamin, New York (1964).

39 A. Karlhede, S. A. Kivelson and S.L. Sondhi, "The Quantum Hall Effect : The article", Lectures pres. at the 9th Jerusalem Winter School on Theor. Phys. (1992).

40 B. I. Halperin, Phys. Rev. B 25, 2185 (1982).

41 J. Voit, Rep. Prog. Phys. 57, 977 (1994); Y. L. Liu, Phys. Rev. B 62, 1212 (2000); X. L. Gao et al., Phys. Rev. A 67, 023610 (2003).

42 F. D. M. Haldane, Phys. Rev. Lett., 51, 605 (1983); F.D.M. Haldane in Ref.[1].

43 I. S. Gradshteyn and I. M. Ryzhik, "Table of Integrals", Academic Dordrecht, New York (1980). 